\title{
CORRECTION
}

\section{Correction to: Prevalence, associated factors and outcomes of pressure injuries in adult intensive care unit patients: the DecubICUs study}

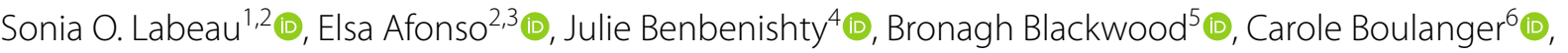

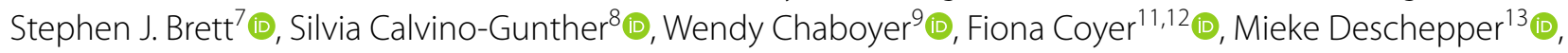 \\ Guy François ${ }^{14}$, Patrick M. Honore ${ }^{15}$ (D), Radmilo Jankovic ${ }^{16}{ }^{\mathbb{C}}$, Ashish K. Khanna ${ }^{17,18}$ (D), Mireia Llaurado-Serra ${ }^{19}$ (D),

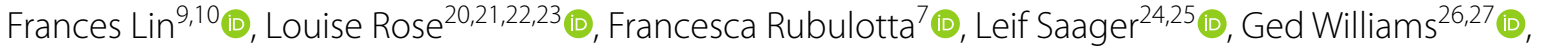 \\ Stijn I. Blot ${ }^{1,2^{*}}(\mathbb{D}$, on behalf of the DecubICUs Study Team and the European Society of Intensive Care Medicine \\ (ESICM) Trials Group Collaborators
}

(c) 2021 The Author(s)

\section{Correction to: Intensive Care Med https://doi.org/10.1007/s00134-020-06234-9}

The original version of this article unfortunately contained a mistake. The members of the ESICM Trials Group Collaborators were not shown in the article but only in the ESM. The full list of collaborators is shown below. The original article has been corrected.

\section{ESICM Trials Group Collaborators}

National Coordinators

Albania: Dritan Muzha (Spitali Universitar i Traumes, Tirana); Andorra: Antoni Margarit Ribas (Hospital Nostra Senyora de Meritxell, Escaldes-Engordany); Argentina: Fernando Lipovesty (Hospital Universitario UAI, Buenos Aires), Cecilia Loudet (General San Martín, La Plata); Australia: Royal Brisbane \& Women's Hospital, Brisbane); Austria: Philipp Eller (Medical University of Graz, Graz); Bangladesh: Nafseen Mostafa (Dhaka

\footnotetext{
*Correspondence: stijn.blot@UGent.be

${ }^{2}$ Department of Internal Medicine, Faculty of Medicine and Health Science, Ghent University, C. Heymanslaan 10, 9000 Ghent, Belgium Full author information is available at the end of the article
}

The original article can be found online at https://doi.org/10.1007/s0013 4-020-06234-9.
Medical College and Hospital, Dakha); Belgium: Patrick M Honoré (CHU Brugmann University Hospital, Brussels); Bolivia: Vanesa Mercado Telleria (Hospital San Juan De Dios, Santa Cruz): Bosnia \& Herzegovina: Jasmina Smajic (University Clinical Center Tuzla, Tuzla); Brazil: Paula Cristina Nogueira (Escola de EnfermagemUniversidade de São Paulo, São Paulo); Brunei: Khalid Mahmood Khan Nafees (RIPAS Hospital, Bandar Seri Begawan); Cameroon: Romuald Hentchoya (Hôpital Général de Douala, Douala); Canada: Louise Rose (Sunnybrook Health Sciences Centre, Toronto); Chile: Javiera Soledad (Clínica Las Condes, Santiago); China: Frances Lin (Griffith University, Brisbane); Colombia: Yenny Cardenas (Fundación Santa F, Bogotá), Amylkar Garay Reyes (Clinica Salud Social, Sincelejo-Sucre); Croatia: Alan Sustic (University of Rijeka Hospital, Rijeka); Cyprus: Meropi Mpouzika (Cyprus University of Technology, Nursing, Limasol); Czech Republic: Tamas Vymazal (Charles University Teaching Hospital Motol, Prague); Denmark: Hanne Irene Jensen (Vejle Hospital, Vejle); Ecuador: Hernan Aguirre-Bermeo (Hospital Vicente Corral Moscoso, Cuenca); Estonia: Liivi Maddison (Tartu University Hospital, Tartu); Finland: Maija Valta (Turku University Hospital, Turku); France: Silvia Calvino-Gunther (Centre Hospitalier Grenoble Alpes, Grenoble); Germany: Frank Bloos (Jena University 
Hospital, Jena); Ghana: Faustina Excel Adipa (School of Peri-Operative and Critical Care Nursing, Korle-Bu, Accra); Greece: Vasilios Koulouras (University Hospital of Ioannina, Ioannina); Honduras: Judy Enamorado (Cardiopulmonary Institute of Honduras); Hungary: Zsuzsann Ágoston (University of Szeged Hospital, Szeged); Iceland: Hrönn Birgisdóttir (Landspitali, Reykjavík); India: Amit Gupta (Bansal Hospital, Bhopal), Mohan Gurjar (S.Gandhi PG Institute of Medical Sciences, Lucknow); Indonesia: Bram Kilapong (Awal Bros Hospital, Batam); Iran: Seyed Mohammadreza Hashemian (Shahid Beheshti University, Tehran); Ireland: Ignacio MartinLoeches (St James's University Hospital, Dublin); Israel: Julie Benbenishty (Hadassah Hebrew University Medical Center, Jerusalem); Italy: Andrea Cortegiani (University Hospital Policlinico P. Giaccone, Palermo); Jamaica: Kelly Fletcher (Hospital of the West Indies, Kingston); Japan: Yoshiro Hayashi (Kameda Medical Center, KamedaHonchō); Kenya: Wangari Waweru-Siika (Aga Khan University Hospital, Nairobi); Republic of Korea: Sang-Min Lee (Seoul National University Hospital, Seoul); Kosovo: Burhan Hadri (University Clinical Centre of Kosova, Prishtinë); Latvia: Mihails Dolgusevs (Riga East University Hospital, Riga); Lebanon: Fayez François Abillama (LAUMCRH, Beirut); Lithuania: Tomas Jovaisa (Barking, Havering and Redbridge University Hospitals NHS Trust, Romford); Luxemburg: Cyril Thix (Hôpitaux Robert Schuman, Luxembourg); Lybia: Muhammed Elhadi (University of Tripoli Hospital, Tripoli); Malaysia: Basri Mat Nor (International Islamic University Malaysia Medical Centre, Kuantan Pahang), Shanti Ratnam (Hospital Sungai Buloh, Sungai Buloh, Selangor), Mohd Zulfakar Mazlan (Hospital Universiti Sains Malaysia, Kota Bharu); Mauritius: Sundaresan Maiyalagan (Wellkin Hospital, Moka, Clinic Muller, Curepipe); Mexico: Luis SánchezHurtado (M. en C. Epidemiología Clínica, Mexico); Moldova: Adrian Belii (State Medical and Pharmaceutical University Nicolae Testemitanu, Chișinău); Mongolia: Mendsaikhan Naranpurev (Mongolian National University of Health Science, Ulaanbaatar); Nepal: Prabha Gautam (TU Teaching Hospital, Kathmandu); Netherlands: Dylan De lange (University Medical Center, Utrecht); New Zealand: Rachael Parke (Auckland City Hospital, Auckland); Nigeria: Rose Ekama Ilesanmi (University of Ibadan, Ibadan); North Macedonia: Mirjana Shosholcheva (Ss Cyril and Methodius University Hospital, Skopje); Norway: Antonija Petosic (Oslo University Hospital, Oslo), Ranveig Lind (The University of Troms $\varnothing-$ The Arctic University of Norway, University Hospital of North Norway, Tromsø); Pakistan: Madiha Hashmi Ffarcsi (Aga Khan University Hospital, Karachi); Paraguay: Javier Bogarin (Osler Clinic, Asunción); Philippines: Aaron Mark Hernandez (Asian Hospital and Medical Center, Muntinlupa); Poland: Malgorzata Mikaszewska-Sokolewicz (Medical University of Warsaw, Warsaw); Portugal: Bruno Sousa (Hospital de Cascais, Cascais); Romania: Dana Tomescu (Institutul Clinic Fundeni, Bucharest), Dorel Sandesc (University of Medicine and Pharmac, Timisoara); Rwanda: Theogene Twagirumugabe (College of Medicine and Health Sciences, Kigali, and University Hospital of Butare); Russia: Vitaly Gusarov (State Budgetary Healthcare Institution, National Medical Surgical Center n.a. N.I. Pirogov, Ministry of Health of Russia, Moscow); Saudi Arabia: Maie Ebaid (Saudi German Hospital, Jeddah); Serbia: Radmilo Jankovic (Clinical Center Nis, Nis); Slovakia: Gari Slobodianiuk, Andrea Martonova (UNB Hospital Ruzinov, $2 / 18$.

Bratislava); Slovenia: Rihard Knafelj (University Medical Center Ljubljana, Ljubljana); South Africa: Mervyn Mer (Charlotte Maxeke Johannesburg Academic Hospital, Johannesburg);Spain: Emilio Maseda (Hospital Universitario La Paz, Madrid); Suriname: Bernardo Panka (s' Lands Hospitaal, Paramaribo); Switzerland: Joerg C. Schefold (Inselspital, Universitätsspital Bern, Bern); Sweden: Eva Joelsson-Alm (Södersjukhuset, Stockholm); Thailand: Konlawij Trongtrakul (Vajira Hospital, Bangkok); Trinidad and Tobago: Lorna Merritt-Charles (Eric Williams Medical Sciences Complex, Champs Fleurs); Tunisia: Lamia Ouanes Besbes (CHU Fatouma Bourguiba, Monastir); Turkey: Yalım Dikmen (Istanbul University-Cerrahpasa, Cerrahpasa School of Medicine); Ukraine: Lesia Zgrzheblovska (Kyiv Emergency Hospital, Kyiv); United Arab Emirates: Mark Fielding (Amana Healthcare Medical and Rehabilitation Hospital, Abu Dahbi); United Kingdom: Francesca Rubulotta (Imperial College NHS, London); United States: Ashish K. Khanna (Wake Forest School of Medicine,Winston-Salem,NC), Leif Saager (University of Michigan, Ann Arbor); Venezuela: Ingrid von der Osten (Hospital Miguel Perez Catteño, Caracas).

\section{Investigators}

ALBANIA: Spitali Universitar i Traumes (Tirana): Dritan Muzha, Alban Greca; University Hospital Shefqet Ndroqi (Tirana): Alma Cani; SUOGJ Koço Gliozheni (Tirana): Nordian Xhindi, Genci Hyska.

ANDORRA: Hospital Nostra Senyora de Meritxell (Escaldes-Engordany): Antonio Margarit Ribas.

ANGOLA: Clínica Multiperfil (Luanda): Susana Pinto, Paulo Alves.

ARGENTINA: Clinica San Camilo (Buenos Aires): Romina Esposito; Emanuel Valgolio; Hospital General de Agudos Dr. Teodoro Alvarez (Buenos Aires): John Thomas Sanchez Minope, Antonio Abdala; Clínica Bazterrica (Buenos Aires): Maria Ayala, Silvina Bravo; 
Sanatorio de la Trinidad Palermo (Buenos Aires): Ana Bantar, Patricia Delgado; Sanatorio Mater Dei (Buenos Aires): Gustavo Badariotti; Hospital Universitario UAI (Buenos Aires): Fernando Lipovestky, Ana Diaz; Policlinico Central Union Obrera Metalurgica (Buenos Aires): Pablo Saul; CEMIC sede Pombo (Buenos Aires): Mariano Setten, Alejandra Aucapina; Sanatorio Sagrado Corazón (Buenos Aires): Ysica Acosta, Victor Gonzalez; Hospital Juan A Fernandez (Buenos Aires): Luis Camputaro, Fernando Baccaro; Obra Social para la Actividad Docente (OSPLAD) (Caba): Robert Villa; Hospital Italiano de Buenos Aires (Caba): Ana Diaz, Marcela Mastantuono; Hospital Justo José de Urquiza (Concepción): Emiliano Dean; Hospital Militar Regional Córdoba (Córdoba): Oscar Fernández Rostello, Patricia Brizuela; Clinica Universitaria Reina Fabiola (Córdoba): Julio Ricardo Bartoli, Matias Guereschi; Hospital Nacional Profesor A. Posadas (El Palomar): Cristian Quiroga, Sofia Putruele; Hospital Francisco Lopez Lima (General Roca, Rio Negro): Paula Villegas, Veronica Curilen; Hospital Español de Mendoza (Godoy Cruz, Mendoza): Ruben Fernandez; Hospital Zonal General de Agudos Simplemente Evita (Gonzalez Catan): Mariangeles Gabriela Nocheretti, Rosana Gabriela Escalante; Hospital General San Martín (La Plata): Cecilia Inés Loudet, Silvia Fernandez; Sanatorio Ipensa (La Plata): Ana Laura Gonzalez; Hospital Dr. Rodolfo Rossi (La Plata): Gustavo Andres Alvarez; Hospital Italiano (La Plata): Federico Iglesias, Silvia Chaparro; Hospital Lagomaggiore (Mendoza): Graciela Zakalik, Gonzalo Pagella; Clínica Privada Monte Grande (Monte Grande): Matías Baini, Pierina Arias Campos; Sanatorio Britanico (Rosario): Ignacio Sabbag, Armando Schmukler; Sanatorio de la Trinidad San Isidro-Sede Thames (San Isidro-Bs. As.): Imelda Perdomo Fonseca, Gonzalo Martín Alvarez; Centro Cuidados Intensivos (San Juan): Mario Ramirez, Fernando Tapia; Hospital Angel Cruz Padilla (San Miguel de Tucuman): Carlos Alejandro Bascary, Graciela del Valle Gimenez; Sanatorio San Jorge (Ushuaia): Fernando Pablo Bertoletti, Esteban Milioto; Clinica San Martin (Villa Maria): Pablo Julio Maldonaldo Bonsignore, Maria Alejandra Fernandez.

AUSTRALIA: Bendigo Hospital (Bendigo): Julie Smith, Tim Chimunda; Sunshine Coast University Hospital (Birtinya): Lorraine Thompson, Teena Maguire; Griffith University (Gold Coast): Frances Lin, Wendy Chaboyer; Royal Brisbane and Women's Hospital (Brisbane): Fiona Coyer, Stacey Watts; Princess Alexandra Hospital (Brisbane): Marion Mitchell, Madeleine Powell; The Prince Charles Hospital (Chermside): India Lye, Leanne Parsons; St Vincent's Hospital (Darlinghurst): Nerilee Baker, Claire Reynolds; Nepean Hospital (Kingswood): Amy Thompson, Kristy Masters; Logan Hospital (Meadowbrook): Kellie Sosnowski, Lynette Morrison; Royal Perth
Hospital (Perth): Gavin D Leslie; Fairfield Hospital (Prairiewood): Ramanathan Lakshmanan; Redcliffe Hospital (Redcliffe): Alexis Tabah, Wendy Brown; Robina Hospital (Robina): Sharon McDowell-Skaines; Rockhampton Hospital (Rockhampton): Andrea McLucas; Gold Coast University Hospital (Southport): Chris Smith, Mandy 3/18.

Tallot; St George Hospital (Sydney): Sarah Jones; Royal Prince Alfred Hospital (Sydney): Michelle Barakat-Johnson, Thomas Leong; The Tweed Hospital (Tweed Heads): Rand Butcher, Kerrie Martin.

AUSTRIA: LKH Univ. Klinikum Graz-Respiratory Care Unit (Graz): Philipp Douschan; LKH Univ. Klinikum Graz-Cardio Intensive Care Unit (Graz): Dirk von Lewinski; LKH Univ. Klinikum Graz-Internal Medicine (Graz): Philipp Eller; Krankenhaus der Barmherzigen Brüder Wien (Vienna): René Schmutz; Landeskrankenhaus Wolfsberg (Wolfsberg): Uta Kolussi;

BAHRAIN: Salmaniya Medical Complex (Manama): Fatema Salman, Zainab Ateya.

BANGLADESH: Dhaka Medical College and Hospital (Dakha): Nafseen Mostafa.

BELGIUM: Onze Lieve Vrouw Ziekenhuis (Aalst): Koen De Decker; ZiekenhuisNetwerkAntwerpen Stuivenberg (Antwerp): Niels Van Regenmortel, Anita Jans, Patricia Wijnands; Imelda Ziekenhuis (Bonheiden): Stefano Coremans; CHU Brugmann (Brussels): Patrick M Honore, David De Bels; Europe Hospitals (Brussels): Tanja Depuydt, Caroline Paillet; UCL-St Luc (Brussels): Luc-Marie Jacquet; AZ Sint-Blasius (Dendermonde): Walter Swinnen; St Dimpna Ziekenhuis (Geel): Francis Hannes, Matthia Mergeay; AZ Maria Middelares (Ghent): Stijn Van de Velde, Silvie Allaert; AZ Sint-Lucas (Ghent): Pieter Hoste, Christophe Borin; CHR Citadelle (Liège): Sandrine Balon, Vincent Fraipont; CHU Charleroi (Lodelinsart): Patrick Biston; Clinique Saint-Pierre (Ottignies): Nicolas De Schryver, Thierry Dugernier; AZ Vesalius (Tongeren): Ilse Van Cotthem.

BOLIVIA: Hospital San Juan de Dios (Santa Cruz): Vanesa Mercado Telleria.

BOSNIA AND HERZEGOVINA: University Clinical Center Tuzla (Tuzla): Jasmina Smajic.

BRAZIL: Hospital de Clínicas da Unicamp (Campinas): Angelica Olivetto de Almeida, Silvia Angelica Jorge; Hospital do Câncer / UOPECCAN (Cascavel): Delmiro Becker, Raysa Cristina Schmidt; Instituto Dante Pazzanese de Cardiologia (São Paulo): Evellyn Oliveira; Sirio Libanes Hospital (São Paulo): Aline Ramalho, Eliane Mazocoli; Hospital do Servidor Público Estadual (São Paulo): Audrey Fioretti, Elaine Barros; Hospital Alemão Oswaldo Cruz (São Paulo): Leticia Serpa, Suzana Bianchini; Instituto do Coração do Hospital das Clínicas da Faculdade de Medicina da Universidade de São Paulo (São Paulo): Ticiane Campanili, Taís Pantaleao; Hospital 
universitário da Universidade de São Paulo (São Paulo): Paulo Carlos Garcia; Hospital Samaritano (São Paulo): Ana Lucia Vitti Ronchini; Instituto Central do Hospital das Clínicas da Faculdade de Medicina da Universidade de São Paulo (ICHC) (São Paulo): Rayanne Santos.

BRUNEI DARUSSALAM: RIPAS Hospital (Bandar Seri Begawan): Khalid Mahmood Khan Nafees, Nurulhuda Binti A. Manap.

CAMEROON: Hôpital Général de Douala (Douala): Romuald Hentchoya.

CANADA: Grey Nuns Community Hospital (Edmonton, AB): Sean Bagshaw, Dominic Carney; Royal Alexandria Hospital (Edmonton, $\mathrm{AB}$ ): Sean Bagshaw, Jon Davidow; Misericordia Community Hospital (Edmonton, AB): Sean Bagshaw, Ella Rokosh; University of Alberta Hospital (Edmonton, AB): Sean Bagshaw; Lachine Hospital McGill University Health Centre (Montreal, QC): Andréa Maria Laizner, Samantha Smith; Montreal Neurological Hospital McGill University Health Centre (Montreal, QC):, Megan McQuirter, Betty Star Kampayana; Royal Victoria Hospital McGill University Health Centre (Montreal, QC): René Favre, Martin Sills; Montreal Chest Institute McGill University Health Centre (Montreal, QC): Andréa Maria Laizner, Julie Dallaire; Montreal General Hospital McGill University Health Centre (Montreal, QC): Andréa Maria Laizner, Cathy Becker; The Ottawa Hospital-General and Civic Campus (Ottawa, ON): Sherissa Microys; University of Ottawa Heart Institute (Ottawa, ON): Bonnie Bowes, Jennifer Lajeunesse; Sault Area Hospital (Sault Ste. Marie, ON): Rishi Ghosh; Scarborough Health Network (Scarborough, ON): Jacqueline Baptiste-Savoie, Rose Raizman; Sturgeon Community Hospital (St. Albert, AB): Sean Bagshaw, Gabriel Suen; Michael Garron Hospital (Toronto, ON): Noushin Taghavi; St. Michael's Hospital (Toronto, ON): Orla Smith; University Health Network (Toronto, ON): Clare Fielding; Sunnybrook Health Science Centre (Toronto, ON): Louise Rose.

CHILE: Guillermo Grant Benavente Hospital (Concepcion): Julieta Canales, Pia Molina; Clinica Las Condes (Santiago): Javiera Chaparro, Maria Idalia Sepulveda; Hospital Felix Bulnes Cerda (Santiago): Matias Jesús Flamm Zamorano, Pamela Rocha; Hospital Clinico General Doctor Raúl Yazigy (Santiago): Ximena Villanueva; Hospital Carlos Van Buren (Valparaíso): Paola Araya; Hospital Naval Almirante Nef (Viña del Mar): Meneses Dayan, Fernando Avalos 4/18.

\section{CHINA:}

Provincial representatives: Liaoning Province: Xiaohan Li, Yu Liu (School of Nursing, China Medical University); Inner Mongolia province: Xinxia Li, Xiaoyan Chen (The Affiliated Hospital of Inner Mongolia Medical University); Guizhou province: Zhixia Jiang, Jing Yang
(Affiliated Hospital of Zunyi Medical College); Guangdong province: Jingfang Chen (Shenzhen third people's hospital); Henan province: Lei Yang (HeNan Honliv Hospital); Shandong province: Kefang Wang, Jie Gao (School of Nursing, Shandong University); Anhui province: Xiuhua Fang, Ronghua Zhao (Anqing Municipal Hospital); Tianjin: Xinhua Xia (TEDA Hospital), Hongmei Liu (Tianjin Third Central Hospital); Beijing: Jing Li (Peking University First Hospital).

Center collaborators: Alxa Central Hospital (Alxa): Haiyan Wang, Gen Meng, Yanhong Di; Anlong County People's Hospital (Anlong, Guizhou): Damei Wang; Anqing Municipal Hospital - site Tian Zhu Shan Dong Road (Anqing): Rong Hua Zhao, Li Ping Hu; Anqing Municipal Hospital - site Renmin Road (Anqing): Xiuhua Fang, Xu Peipei; Anqing First People's Hospital (Anqing): Qing Feng Jiao; Susong County People's Hospital (Anqing): Hai Yun Wang; Marine Anqing Hospital (Anqing): Chun Jie Xia; Anshan Central Hospital (Anshan): Yan Liu; Anshun Shirenminyiyuan (Anshun): Mei Ye; Hospital 302 Attached to Guiyang Aviation Group (Anshun): Yan Wan; The First Affiliated Hospital of Baotou Medical College of Inner Monglia University of Science and Technology (Baotou): Wenmei Wang; Inner Mongolia Baogang Hospital (Baotou): Yajun Ding; BAYANUR Hospital (Bayannaoer): Aiua Ren; Dengkou County Hospital (Bayannaoer): Yan Gao; Cancer Hospital Chinese Academy of Medical Sciences (Beijing): Qi Li;; Capital Medical University Beijing Anzhen Hospital ICU (Beijing): Guifang Du; China-Japan Friendship Hospital MICU (Beijing): Jing Yang; China-Japan Friendship Hospital ICU (Beijing): Yanling Shen; Peking University First Hospital EICU (Beijing): Yanming Ding, Ning Li; Peking University First Hospital SICU (Beijing): Cui Yuan,Jing Li; Beijing Jiangong Hospital (Beijing): Lei Tan, Qiang Lin; Peking Union Medical College Hospital (Beijing): Hailing Guo; Beijing Ji Shui Tan Hospital EICU (Beijing): Howe Yan; Beijing Ji Shui Tan Hospital ICU (Beijing): Xiao Xu; FuXing Hospital,Capital Medical University ICU (Beijing): Wei Zhang; The 302 Hospital of PLA (Beijing): Jinxian Liang, Libing Zhang; Armed Police General Hospital (Beijing): Eryun Tian, Qian Zhao; Aerospace Central Hospital (Beijing): Lin InSu, Jingwen Dong; Capital Medical University Affliated Beijing You an Hospital (Beijing): Yanmei Gu; China Academy of Chinese Medical WangJing Hospital (Beijing): Ying Liu, Lina Zhao; Beijing Cancer Hospital (Beijing): Wei Wang; Peking University Third Hospital (Beijing) RICU: Hongmei Qiao, Lili Tuo, Mengmeng Lv; Peking University Third Hospital YanQing Hospital (Beijing): Jin Yu Zhu, JiFen Zhu; LiangXiang Hospital ICU (Beijing): Ying Wei, Man Liu; Capital Medical University Tongren Hospital ICU(Beijing): Yin He; Peking University First 
Hospital CCU (Beijing): Jiying Cheng, Jin Liu; Peking University First Hospital RICU(Beijing): Na Jia; Peking University First Hospital Geriatric ICU(Beijing): Dongfang Wei; Peking University People's Hospital (Beijing): Qing Li, Xiaoying Wu; The First Affiliated Hospital of Bengbu Medical College (Bengbu): Huan Duan, Dongliang Lin; The People's Hospital of Weining Autonomous County (Bijie): Qiong Liang; Qianxi County People's Hospital (Bijie): Xiaofang Luo, Yunfeng Xiong; First People's Hospital of Bijie Cit (Bijie): Rong Fen Huang, Jing Fu; The First Hospital of Jilin University (Changchun): Tao Zan; The Second Xiangya Hospital, Central South University (Changsha): Man Ye; Hunan Provincial People's Hospital / The First Affiliated Hospital of Hunan Normal University (Changsha): Zeya Shi, Yanfei Long; HeNan Honliv Hospital (Changyuan): Yang Lei; Chaoyang Central Hospital (Chaoyang): Xiaodi Liu; Sichuan Provincial Biyi Rehabilitation Center (Chengdu): Chen Yumei; Chifeng Municipal Hospital (Chifeng): LingLing Wang,; Chifeng Institute Affiliated Hospital (Chifeng): Yali Zhang, Yan $\mathrm{Xu}$; Chizhou People's Hospital of Anhui (Chizhou): Cheng; Qianjiang Center Hospital (Chongqing): Wang Zhijuan; The Second Hospital of DaLian Medical University (Dalian): Chengxia Sun; Zhongshan Affiliated Hospital of Dalian University SICU (Dalian): JinHui Song; Zhongshan Affiliated Hospital of Dalian University EICU (Dalian): Yingli Wang; The First Affiliated Hospital of Dalian Medical University (Dalian): XiuMei Liu; Dalian Beihai Hospital (Dalian): Yupeng Liu; Central Hospital Dandong (Dandong): Yuxia Yuan; Dongguan Houjie Hospital (Dong Guan): Qingping Huang, Fengling Yang; Third Affilia Hospital of GuiZhou Medical University (DuYun): Yun Wu; The People 's Hospital of Guiding County (Duyun): Xianping Luo; GuiZhou QianNan People's Hospital (Duyun): Xiaowu Bai, Hong Zheng; Fushun Mining Bureau General Hospital Internal Medicine ICU (Fushun): Min Song; Fushun Mining Bureau General Hospital Department Critical Care (Fushun): Yue Sun; Affiliated Hospital of Guizhou Medical University (Guiyang): ZhangShuangzi Li; Xiaohe River 44 Hospital (Guiyang): Feifei Luo; Affiliated Wudang hospital of Guizhou Medical University (Guiyang): Miao Liu; First people's Hospital of Guiyang (Guiyang): Li Chuntang Li, Xinjian Li; People's Hospital of Guizhou Province (Guiyang): Guiping Zhang; Second Affiliated Hospital of GuiYang Medical College (Guiyang): Lina Xiao, Tingting Yu; Guizhou Cancer Hospital (Guiyang): Guangyuan Gao, Wei Wei; 5/18.

Jinyang Hospital in Guiyang (Guiyang): Fanglan Wang, Ting Han; Zhijin County People's Hospital (Guizhou): Tingting Li, Qi Zeng; Liupanshui Maternal and Child Healthcare Hospital, (Guizhou): Jing Mei Zeng; Songtao Miao Autonomous County People Hospital of teaditional
Chinese Medicine National Hospital of Traditional Chinese Medicine (Guizhou): Yan Long; People's Hospital of Danzhai County (Guizhou): Fuqun Pan; People's Hospital of Dafang (Guizhou): Jing Wang, Guoxue He; Majiang County People's Hospital (Guizhou): Haiyan Chen, Feifei Zhang; First Hospital Affiliated of Medical College of Zhejiang University (Hang Zhou): Yu Chao, Gao Chunhua; The First Affiliated Hospital of Universty of Science and Technology of China (Hefei): Xiuying Yao; The First Affiliated Hospital of Anhui University of Traditional Chinese Medicine (Hefei): Dongmei Bai, Lu Liu; The Inner Mongolia Autonomous Region People's Hospital (Hohhot): Xuelian Xu; The Second Affiliated Hospital of Inner Mongolia Medical University (Hohhot): Yan Wang; Hohhot First Hospital (Hohhot): Xuejuan Liang; Inner Mongolia International Mongolia Hospital (Hohhot): Na Zhang; The Affiliated Hospital of Inner Mongonlia Medical University (Hohhot):Xinxia Li; The Affiliated Hospital of Inner Mongonlia Medical University ICU (Hohhot):Aizhi Zhang, Xiaoyan Chen; The Affiliated Hospital of Inner Mongolia Medical University RICU (Hohhot): XiaoChun Hu; The Affiliated Hospital of Inner Mongolia Medical University EICU (Hohhot): Hui Zhang; The Affiliated Hospital of Inner Mongolia Medical University NICU (Hohhot): RuiXia Wang; Prince of Wales Hospital (Hong Kong): Poon Shing Tak, Sung Wai Ho; Huaining County People's Hospital (Huaining): Qun Xia Jiang; Huludao Central Hospital-Lianshan Area (Huludao): Xinran Ding; Huludao Central HospitalLonggan Area (Huludao): Liu Hong; Hulunbuir People's Hospital (Hulunbair): Limei Miao;Inner Mongolia Forestry General hospital: Zhaoxia Feng; JiangYin people's Hospital (JiangYin): LiPing Huang; Jianhe County People's Hospital (Jianhe): Juan Wu; Shandong Provincial Hospital ICU (Jinan): Yuping Wang, Jiye Guo; Shandong Provincial Hospital EICU (Jinan): Baoke Zhang, Chaoqun Ma; Shandong Provincial Hospital SICU (Jinan): Yu Han, Congcong Liu; Shandong Provincial Hospital MICU (Jinan): Min Ding, Linlin Luan; Shandong Provincial Hospital CSICU(Jinan): Jing Zheng, Shanshan Lv; Shandong Provincial Qianfoshan Hospital ICU1 (Jinan): Shumin Jiang, Wenzhen Cao; Shandong Provincial Qianfoshan Hospital ICU2 (Jinan): Xiujuan Xue; Qilu Hospital of Shandong University CCU(Jinan): Jing Li, Guangyan Liu; Qilu Hospital of Shandong University ICU1 (Jinan): Jing Wang, Xiyan Wei; Qilu Hospital of Shandong University ICU2 (Jinan): Wei Zhang, Youru Jiang; The Eastern Hospital ICU of Qilu Hospital (Jianan): Zhiru Yao, Li Gao; Qilu Hospital of Shandong University CSICU(Jinan): Jinhua Li, Wenwen Zhao; Qilu Hospital of Shandong University EICU (Jinan): Mei Jiang; Qilu Hospital of Shandong University RICU (Jinan): Junping Hao, Jing Zhang; The Second Hospital of Shandong University 
GICU (Jinan): Caiju Song, Feifei Chen; The Second Hospital of Shandong University Neurology ICU1(Jinan): Shuhui Wang, Lili Hu; The Second Hospital of Shandong University Neurology ICU2(Jinan): Deyan Cao, Yan Liu; The Second Hospital of Shandong University CSICU (Jinan): Jianhong Wan, Xiaomin Wang; The Second Hospital of Shandong University RICU (Jinan): Hongyan Shao, Zhenxia Zhang; The Second Hospital of Shandong University NSICU(Jinan): Xia Cui, Jingyu Liu; The Second Hospital of Shandong University EICU(Jinan): Lijuan Zhao, Xingguo Li; Jingjiang Pepole's Hospital (Jingjiang): Limei Fan; The First People's Hospital of Jing Zhou (Jingzhou): Ling Zhang; Qiandongnan People's Hospital (Kaili): Min Yu, Biyan Li; Second Affiliated Hospital of Guizhou Medical University (Kaili): Chunxia Li, Ling Liu; Yan'an Hospital of Kunming City CSICU (Kunming): XueLian Liu, Wenmin Chen; Yan'an hospital of Kunming City ICU (Kunming): Yan Li; First Hospital Affiliated to Lanzhou University (Lanzhou): Zhang Zhigang, Wu Yuchen; Second Hospital Affiliated to Lanzhou University (Lanzhou): Chenghau Mu; Liupanshui People's Hospital of Guizhou Province (Liupanshui): Guoyan Zhu, Fan Yang; Qibo Hospital (Long Jiang): Qi Bo; Nanjing Tongren Hospital (Nanjing): Ling Li; Erdos Central Hospital (Erdos): Meili Chen; Qianshan County Hospital (Qianshan Province, Anqing): Jing Hua Jiang; Qilu Hospital of Shandong University (Qingdao): Hai Yin, Xuelian Pang; Qinghai Provincial People's Hospital (Qinghai): Yue Ying Gong; Qian Nan Zhou Zhong Yi Yi Yuan (Qui Yang): Shunzhu Yang; Quzhou Kecheng Hospital (Quzhou): Xiaoli Yan, Xianhong Zheng; RenHuai Municipal Hospital or TCM (Renhuai): DeHong Lei; Renhuai People's Hospital (Renhuai): Lei Lei; ShengJing Hospital of China Medical University (Shengyang): Yinhua Guo; The Fourth Affiliated Hospital of China Medical University (Shengyang): Lihong Liu; The 4th people's Hospital of Shengyang (Shengyang): Jing Yu, Wei Sun; Shenyang Medical College Affiliated Central Hospital RICU (Shenyang): AiPing Bi; Shenyang Medical College Affiliated Central Hospital Dept of Critical Care (Shenyang): Weiwei Li; Liaoning People's Hospital (Shenyang): Yang Wu; Shenyang Medical College Affiliated Central Hospital Severe Infections Care Unit (Shenyang): Ji Li; The First Affiliated Hospital of China Medical University (Shenyang): Dongshu Ni; China Medical University School of Nursing (Shenyang): Xiaohan Li, Yu Liu, Zijing Wu, Bing Song; The Third People's Hospital of Shenzhen (Shenzhen): Jingfang Chen, Qin Fei; First Affiliated Hospital Shihezi University (Shihezi): Yang Xiaoyan; Da Zhou Central Hospital (Sichuan): Qiong Ran; Suining Central Hospital (Suining): Li 6/18.

Xixi; Shanxi Provincial Cancer Hospital (Taiyuan): Xueping Jiao, Hua Ji; Tianjin Academy of Traditional
Chinese Medicine Affiliated Hospital (Tianjin): Sun Zhiping; Tianjin People's Hospital (Tianjin): Ma Hong, Mu Jianhong; Tianjin Third Central Hospital (Tianjin): Yanhua Hao, Li Yin; The First Central Hospital of Tianjin (Tianjin): Ying Wang, Caihong Hui; Tianjin Yikedaxue Dieryiyuan (Tianjin): Wenjie Ju; Tianjin TEDA Hospital(Tianjin): Xinhua Xia; Tianjin TEDA Hospital NICU (Tianjin): Yuxia Huo; Tianjin TEDA Hospital ICU (Tianjin): Yuxia Wang; Tianjin Medical University Cancer Hospital (Tianjin): Lei Chen; First Teaching Hospital of Tianjin University of TCM (Tianjin): Yan Yan; Tianjin Baodi People's Hospital (Tianjin): Qingli Zhao; PLA 464 Hospital (Tianjin): Hongjuan Chen; Tianjin Haihe Hospital (Tianjin): Guijun Bao; The Fourth Center Hospital of TianJin (Tianjin): Ying Cao; Tianjin Huanhu Hospital (Tianjin): Li Hong; Tianjin NanKai hospital (Tianjin): Hong Zhang; TianJin Haibin People's Hospital (Tianjin): Ying Zhang; Tianjin Chest Hospital (Tianjin): Lina Xu; Tianjin Shijizhou Qurenminyiyuan (Tianjin): Jia Guixiang; Tianjin Hospital (Tianjin): Ying Li; Tianjin Jinnan District Xian Shui Gu Hospital (Tianjin): Hui Min Zhao; Tongren Shi Ren Min Hospital (Tongren): Xia Huang, Zhaoxing Dai; The People's Hospital of The Jiang County (Tongren): Yanman Jian, Hongsu Zhang; YanHe County People's Hospital (Tongren): Zhixia Tian; Wang Jiang County People's Hospital (Wang Jiang): Zu Qing Cao; Weinan Central Hospital (Weinan): Miao Li, Yang Liu; Wuhan Fourth Hospital, Tongji Medical College, Huazhong University of Science \& Technology (Wuhan): Fei Ouyang; XiaMen University Affiliated ZhongShan Hospital (XiaMen): Fuying Ma; Xiaoshan First People's Hospital (Xiaoshan): Wangyan Jin, Liuyan Ge; Xifeng Conuty Hospital of traditional Chinese Medicine (Xifeng): ShiFen Wu; People's Hospital of Zhenfeng County (Xingyi): Jing Li, Weilian Yuan; Qianxinan People's Hospital (Xingyi): Tianfei Chen, Guanxiu Shi; Qianxinan Orthopedic Hospital (Xingyi): Zhihong Chen, Kewei Liu; Xingyi People's Hospital of Guizhou (Xingyi): Xue Lin; Qinghai Provincial People's Hospital (Xining): Ly Yuemen, Sun Lijuan; Xiuwen County People's Hospital (Xiuwen): Xiao Fang Tian; Xuanwu Hospital (Xuanwu): Shuo Wang; Inner Mongolia Forestry Feneral Hospital (Yakeshi city of Hulun Buir League): ZhangXia Feng, XiaoZhe Liu; Yuxi People's Hospital of Yunnan Province (Yuxi): Yunchun Dong; China tianjin shixiqing hospital (Tianjin): Jundi Zhang; Linzi District People's Hospital (Zibo): Nie Bocui; Affiliated Hospital of Zunyi Medical College (Zunyi): ZhiXia Jiang, Jing Yang; The First people's Hospital of Zunyi (Zunyi): GuoXian Wang; The Traditional Chinese Medicine Hospital of Zunyi (Zunyi): Yingjuan Zhao; People's Hospital of Zunyi City Bo Zhou District (Zunyi): Xiaojun Wu; TongZi People's Hospital (Zunyi): Qiao Yang, Rongjun Ling Hu; Suiyang People's 
Hospital (Zunyi): Xue Qin Li, Zhu Jun Yu; Daozhen Autonomous County People's Hospital (Zunyi): Yanlan Yao; Gguizhou Province Xishui County People`s Hospital (Zunyi): Xiaoqiong Deng; MeiTan People's Hospital (Zunyi): Yan Xiao, Yan Xie; The People's Hospital of Zhengan County (Zunyi): Yanping Yang; Yuqing Xian Renming Yiyuan (Zunyi): Huai Yang, Yuming Zhou; People's Hospital of Honghuagang District (Zunyi): Zhuqing Li; People's Hospital of Fenggang County (Zunyi): Min Xiao, YongXia Yang; Wuchuan County People's Hospital (Zunyi): Yani Tian;

COLOMBIA: Fundación Hospital San Carlos (Bogota): Luz Marina Silva Gama, Juan Sebastian Hernandez; Hospital Universitario Fundación Santa Fe de Bogotá (Bogota): Yenny Cardenas; Clínica San Marcel (Manizales): Nestor Caicedo, Jorge Marin; Fundacion Valle Del Lili (Cali): Maria-Elena Ochoa, Monica Gomez; Gestión Salud IPS (Cartagena): José RojasSuarez, Jeniffer Gonzalez; Clinica Salud Social (Sincelejo-Sucre): Amylkar José Garay Reyes; IPS Arcasalud (Zipaquira): Edwin Chapeta, Estefania Orozco.

CROATIA: European Medical Center-General Hospital Dubrovnik (Dubrovnik): Ina Filipović-Grčić, Anita Vuković; Pula General Hospital (Pula): Suzana Pečenković, Aleksandar Šuput; KBC Rijeka (Rijeka): Alan Sustic; General Hospital Dr Ivo Pedisic (Sisak): Gordana Zivanovic-Posilovic; Klinički Bolnički Centar (Split): Armanda Bozena, Nikolina Udiljak; University Hospital Dubrava (Zagreb): Morena Milic; University Hospital Centre Zagreb SICU (Zagreb): Renata Curic Radivojevic, Slobodan Mihaljevic; University Hospital Centre Zagreb NIC (Zagreb): Marijana Matas, Dinko Tonkovic; University Hospital Centre Zagreb CICU (Zagreb): Hemena Čuljak, Ivana Herceg; University Hospital Centre Zagreb RICU (Zagreb): Gordana Pavlisa; Traumatology Clinic, Clinical Hospital Centre Sestre Milosrdnice (Zagreb): Milena Dobric, Tatjana Beker; Clinical Hospital Sveti Duh (Zagreb): Višnja Nesek Adam, Tanja Goranovic.

CYPRUS: Larnaca General Hospital (Larnaca): Chrysanthos Markoulias, Mina Mathaios; YGIA Polyclinic (Limasol): Maria Mylordou; Limasol General Hospital (Limasol): Eleni Achilleos, Pavlina Kleanthous; Mediterranean Hospital of Cyprus (Limasol): Veronika Kotanidi; Nicosia General Hospital (Nicosia): Maria Foka, Iwy Charalabous; American Medical Center (Nicosia): Anna Alexandrou, Marios Georgiou; Aretaeio Private Hospital (Nicosia): Artemis Patsalos; Apollonio Private Hospital Nicosia (Nicosia): Sofia Zepoy; Paphos General Hospital (Paphos): Constantina Constantinou 7/18.

CZECH REPUBLIC: IKEM (Prague): Petr Piza; Motol University Hospital (Prague): Tomas Vymazal.
DENMARK: Sygehus Sønderjylland (Aabenraa): Elisabeth Wiborg, Louise Bruhn; Aalborg University Hospital (Aalborg): Karin Kaasby, Karin Rehnholt Pedersen; Aarhus University Hospital (Aarhus): Sanne Mikkelsen; Rigshospitalet University Hospital ICU (Copenhagen): Marie Collet; Rigshospitalet University Hospital Thorax ICU (Copenhagen): Anne Langvad, Hanne Andresen; Sydvestjysk Sygehus (Esbjerg): Susanne Fischer, Inger Ebbesen Kjærgård; Gentofte Hospital (Hellerup): Britta Jepsen; Regionshospitalet Herning (Herning): Birthe Husted; Nordsjællands Hospital (Hillerød): Morten Bestle, Anne Marie Kodal; Holbæk Sygehus (Holbæk): Tina Charlotte Bitsch Hansen; Horsens Regional Hospital (Horsens): Anne Sofie Bomholt Pedersen; Kolding Sygehus (Kolding): Tina Damgaar Thomsen; Regionshospitalet Randers (Randers): Anisette Hoegenhaven; Regionshospitalet Silkeborg HE-midt (Silkeborg): Mette From, Tine Melgaard Frandsen; Sygehus Sønderjylland (Sønderborg): Grit Henning, Anja Hansen; Vejle Hospital (Vejle): Hanne Irene Jensen; Inger Abildgaard Bliksted.

ECUADOR: Hospital Santa Ines (Cuenca): Luis Mario Tamayo, Pedro Mogrovejo; Hospital Vicente Corral Moscoso (Cuenca): Hernan Aguirre-Bermeo, Carolina Palaez; Hospital de Especialidades Eugenio Espejo (Quito): Diego Rolando Morocho Tutillo, Cintia Valencia Hurtado; Clínica la Merced (Quito): Maria Fernanda García, Diana Alvarez; Hospital Carlos Andrade Marin (Quito): Fausto Guerrero, Alexandra Vasquez.

ESTONIA: North Estonia Medical Centre Foundation (Tallinn): Martin Kütimets; Tartu University Hospital ICU1 (Tartu): Kadri Tamme; Tartu University Hospital ICU2 (Tartu): Liivi Maddison; Tartu University Hospital ICU3 (Tartu): Eneli Anvelt.

KINGDOM OF ESWATINI: Mbabane Government Hospital (Mbabane): Lomangisi Dlamini-Sserumaga.

FINLAND: Helsinki University Hospital Töölön tehoja tehovalvontaosastob - M20 (Helsinki): Carita Löfqvist; Helsinki University Hospital Teho- ja tehovalvontaosasto M1 (Helsinki): Virpi Lusenius; Helsinki University Hospital Palovammakeskus ja tehohoito U2 (Helsinki): Outi Kauppi; Helsinki University Hospital Cardiac Surgical ICU M2A (Helsinki): Jenni-Katarina Sakki; Kuopio University Hospital (Kuopio): Tarja Tervo-Heikkinen, Ulla Kesti; Oulu University Hospital (Oulu): Merja Merilainen, Elina Karjula; Tampere University Hospital (Tampere): Minna Peltomaa, Auli Palmu; Turku University Hospital (Turku): Maarit Ahtiala, Maija Anniina Valta.

FRANCE: Hôpital Victor Dupouy (Argenteuil): Hervé Mentec, Gaëtan Plantefève; CHU Besançon (Besançon): Guillaume Besch, Sébastien Pili-Floury; Centre Hospitalier Pierre Oudot (Bourgoin Jallieu): Stanislas Ledochowski; Hôpital d'Instruction des Armées Clermont 
Tonnerre (Brest): Marc Danguy des Déserts, Christophe Giacardi; CHU Caen (Caen): Cédric Daubin; CHUR Dijon-Bourgogne (Dijon): Audrey Massard; Grenoble Alpes University Hospital-Rea CVT (Grenoble): Yann Le Guen; Grenoble Alpes University Hospital-RCP (Grenoble): Agnès Blanc; Grenoble Alpes University HospitalRea Neurochirugicals (Grenoble): Simon Mandaroux; Grenoble Alpes University Hospital-MICU (Grenoble): Silvia Calvino Günther, Prune Avogadro; Hôpital Militaire Desgenettes (Lyon): Anthony Radavidson, Jean Turc; Groupe Hospitalier Sud Ile-de-France, Hôpital de Melun (Melun): Sébastien Jochmans; CHU Nice (Nice): Hervé Quintard, Laetitia Boyer; Groupe Hospitalier Paris Saint Joseph (Paris): Cédric Bruel, François Philippart; Hôpital Bichat -Claude Bernard (Paris): Philippe Montravers, Enora Atchade; Groupe Hospitalier Pitié Salpêtrière (Paris): Nadine Flessel, Benoît Chinardet; Hôpital Lariboisière (Paris): Léa Soulisse, Cindy Pillard; GH Saint Louis-Lariboisière-Fernand Vidal-Centre de Traitement des Brûlés (Paris): Delphine Ngo; GH Saint Louis-Lariboisière-Fernand Vidal-Réanimation Chirurgicale Polyvalente (Paris): Benjamin Bongiorno, Nathalie Heitzler; GH Saint Louis-Lariboisière-Fernand Vidal Réanimation Médicale (Paris): Virginie Souppart, Nathalie Gautheret; Hôpital Bichat (Paris): Jean-Francois Timsit, Fatiha Essardy; APHP Tenon (Paris): Muriel Fartoukh, Daisy Mehay; CHU Poitiers (Poitiers): Fabienne Etourneau; Polyclinique Saint André (Reims): Jean-christophe Farkas; Centre Hospitalier Roanne (Roanne): Pascal Beuret; Hôpital Delafontaine (Saint Denis): Gabriel Preda, Etienne De Montmollin; Hôpitaux Universitaires de Strasbourg-Hôpital de Hautepierre (Strasbourg): Vincent Castelain.

GERMANY: Universitätsklinikum Augsburg (Augsburg): Ulrich Jaschinski, Monika Rothenfusser; University Hospital Duesseldorf (Duesseldorf): Detlef KindgenMilles, Thomas Dimski; Universitätsklinikum Erlangen (Erlangen): Christine Fiedler, Tobias Heinicke; University Hospital Frankfurt (Frankfurt): Patrick Meybohm; Universitätsmedizin Greifswald (Greifswald): Tobias Schulze; Bethesda Krankenhaus Bergedorf (Hamburg): Marc Bota; BG Klinikum Hamburg (Hmaburg): Sabrina Pelz, Tobias Odenthal; Marien Hospital Herne (Herne): Martin Christ; Jena University Hospital (Jena): 8/18.

Frank Bloos; VAMED Klinik Kipfenberg (Kipfenberg): Kathrin Bösl, Achilleas Chovas; University Hospital of Leipzig (Leipzig): Sebastian Stehr, Philipp Simon; Klinikum rechts der Isar der TUM IS1 (Munich): Sarah Grotheer, Sebastian Schüppel; Klinikum rechts der Isar der TUM IS2 (Munich): Stefan Schaller, Lea Albrecht; Klinikum rechts der Isar der TUM ISR3a (Munich): Andeas Stübner, Stephan Graeser; Münster University Hospital (Muenster): Nina Kolbe, Martina Lausch;
Universitätsklinik f. AINS, Klinikum Oldenburg (Oldenburg): Anja Diers, Ulf Guenther; University Hospital Tübingen (Tuebingen): Reimer Riessen, Martin Roller.

GHANA: Cape Coast Teaching Hospital (Cape Coast): Irene Pearl Osei; Komfo Anokye Teaching Hospital (Kumasi): Anita-Chrysolyte Kusi-Appiah; Tamale Teaching Hospital (Tamale): Yakubu H. Yakubu; Tema General Hospital (Tema): Belinda Guadi-Gosh.

GREECE: University General Hospital of Alexandroupolis (Alexandroupolis): Christos Dragoumanis, Christos Christofis; General Hospital of Arta (Arta): Nikolaos Kazakos, Styliani Bastani; Naval Hospital (Athens): Charalampos Martinos, Vasileios Bekos; Hippocration General Hospital of Athens (Athens): Metaxia Papanikolaou, Theonymfi Papavasilopoulou; Saint Savvas Hospital (Athens): Anna Efthymiou, Vasiliki Chantziara; Sotiria Chest Diseases Hospital (Athens): Anna Kyriakoudi, Nikolaos Kakaras; Attikon University Hospital (Athens): Chrisi Diakaki, Aikaterini Flevari; Konsantopoulion - Patission G.H (Athens): Charikleia Nikolaou, Kounougeri Katerina; GNA G. Gennimatas Hospital (Athens): Lamprini Avramopoulou, Kyriaki Tsikritsaki; Aretaieion University Hospital (Athens): Georgios Gkiokas, Eirini Pantiora; Korgialenio-Benakio Red Cross Hospital (Athens): Chrysostomos Katsenos, Eirini-Chysovalanto Patsiou; General Hospital of Elefsis (Athens): Paraskevi Alexandropoulou, Ioannis Koutsodimitropoulos; Corfu General Hospital (Corfu): Epaminontas Farmakis, Konstantina Nestora; Mediterraneo Hospital (Glyfada): Marinos Chatzis; University Hospital of Heraklion (Heraklion): Eumorfia Kondili, Stella Soundoulounaki; G.H.'G.Chatzikosta (Ioannina): Ourania Mousafiri, Dimitra Lepida; University Hospital of Ioannina-Cardiotheracic Postoperative ICU (Ioannina): Antonia Liarmakopoulou; University Hospital of Ioannina ICU (Ioannina): Vasilios Koulouras, Georgios Papathanakos; General Hospital Katerini (Katerini): Mrina Oikonomou, Panagiotis Ioannides; General Hospital of Larissa (Larissa): Dimitrios Papadopoulos, Ioannis Staikos; Sismanoglio General Hospital (Marousi): Maria Stafylaraki; Sismanoglio-A. Fleming Gen. Hosp. Attika (Marousi): Bogdan Raitsiou, Konstantinos Mandis; General Hospital of Patras Agios Andreas (Patras): Ifigenia Ravani, Styliani Kourelea; AHEPA University General Hospital (Thessaloniki): Aikaterini Efthimiou, Giannoula Thoma; General Hospital O Agios Dimitrios (Thessaloniki): Apostolos Bakas, Konstantinos Psarulis; Theagenion Hospital (Thessaloniki): Souzana Anisoglou, Eirini Papageorgiou; G.H.T.Ippokrateio (Thessaloniki): Evangelia Michailidou, Thomai Tholioti; Papanikolaou Hospital (Thessaloniki): Athena Lavrentieva, Evdokia Sourla; General Hospital of Tripolis-Panarcadian (Tripolis): Anastasia Spyropoulou, Nikolaos Pantelas; GH Imathia Veria 
(Veria): Kristina Mariana Matei Stalika, Ioannis Georgakas; G.H.Volos Achillopouleio (Volos): Antigoni Karathanou, Syragoula Tsikriki; Asklepieion Voulas General Hospital (Voula): Aikaterini Dimoula, Sofia Kanakaki; Xanthi General Hospital (Xanthi): Aristeidis Vakalos, Konstantinos Pagioulas.

HONDURAS: Instituto Nacional Cardiopulmonar (Tegucigalpa): Judy Enamorado Enamorado,

HUNGARY: Péterfy Sándor utcai Kórház és Baleseti Központ (Budapest): Gabor Nardai; University Hospital of Szeged (Szeged): Fatime Hawchar.

ICELAND: Sjukrahusid Akureyri (Akureyri): Asbjorn Blondal, Brynja Rygvadottir; Landspitali-National University Hospital of Iceland ICU12B Hringbraut (Reykjavik): Rannveig J. Jonasdottir; Landspitali-National University Hospital of Iceland E6 Fossvogur (Reykjavik): Hrönn Birgisdottir.

INDIA: CIMS Hospital (Ahmedabad): Bhagyesh Shah, Shuchi Kaushik; All India Institute of Medical Sciences (Bhubaneswar): Swagata Tripathy; Himalayan Institute of Medical Sciences (Dehradoon): Mukta Singh, Sonika Agarwal; Max Super Specialty Hospital (Ghaziabad): Manish Gupta, Meraj Ahmad; Eternal Hospital (Jaipur): Kishore Mangal, Vaibhav Bhargava; Apollo Hospital (Nashik): Vilas Kushare; PSRI Hospital (Delhi): Simant Jha, Lakshay Bhakhtiani; Bansal Hospital (Bhopal): Amit Gupta; AIIMS Jodhpur (Jodhpur): Manoj Kamal; Sanjay Gandhi Postgraduate Institute of Medical Sciences (SGPGIMS) (Lucknow): Mohan Gurjar, Arvind Baronia.

INDONESIA: Awal Bros Hospital (Batam): Bram Kilapong; Rsud Raden Matthaher Jambi (Jambi): Ade Susanti; Mohammad Hoesin Hospital (Palembang): Mayang Indah Lestari, Zulkifli Zulkifli; Rumah Sakit Agung (South Jakarta): Windu Baskoro 9/18.

IRAN: Nemazee Hospital ACCRC (Shiraz): Farid Zand, Fatemeh Zarei; Imam Reza Hospital (Tabriz): Ata Mahmoodpoor; Milad General Hospital (Tehran): Farshad Heidari; Poursina Hospital (Rasht Guilan): Fateme Jafaraghaee.

IRELAND: Mayo University Hospital (Curragh,Castlebar): Aidan O'Shea, Fiona O'Shea; Our Lady of Lourdes Hospital (Drogheda): Caroline O' Donnell, Geraldine Craig; Tallaght Hospital (Dublin): Gerry Fitzpatrick, Lisa Dunne; Mater Misericordiae University Hospital (Dublin): Jennifer Hastings, Brian Marsh; Connolly Hospital (Dublin): Caitriona Cody, Elizabeth Campbell; St James's Hospital (Dublin): Deirdre Doyle, Michelle Pacturanan; University Hospital Galway (Galway): Christine Sheehan, Annette Carey; Portiuncula Hospital (Galway): Charlotte Carter, Regina Mulvey; Letterkenny University Hospital (Letterkenny): Damien O'Connell Rosemary Finn; University Hospital Limerick (Limerick): Catherine Motherway, Amy Walsh; Naas
General Hospital (Naas): Jennifer Kehoe, Shella Delossantos; Midland Regional Hospital Portlaoise (Portlaoise): Jennifer Lalor; University Hospital Kerry (Tralee): Siobhan O'Nuallain, Helena Behan; University Hospital Waterford (Waterford): Sandra McPherson, Ailesh Corcoran; Wexford General Hospital (Wexford): Patricia Gordon, Glenda Rooney.

ISRAEL: Rambam Health Care Campus (Haifa): Dassy Levy; Carmel Medical Center (Haifa): Mazal Azencot; Hadassah Hebrew University Medical Center (Jerusalem): Julie Benbenishty; Assaf Harofeh Medical Center (Zerifin): Vladimir Gurevich, Alinoy Lavy.

ITALY: Azienda Ospedaliera Universitaria OORR di Ancona (Ancona): Valentina Bendelari, Romina Marconi; A.O.U. Ospedali Riuniti di Ancona-UTIC (Ancona): Antonio Barone, Chiara Gatti; A.O.U. Ospedali Riuniti di Ancona - Rianimazione (Ancona): Andrea Giampaoletti, Cinzia Borgognoni; Humanitas Mater Domini (Castellanza): Davide Massimo Ghioldi; Azienda Ospedaliera Garibaldi Nesima (Catania): Arena Raimondo; Ospedale Vittorio Emanuele (Catania): Giacomo Castiglione, Anna Vita Bruno; Azienda Ospedaliera per l'Emergenza Cannizzaro-Terepia Intensive Trauma Center (Catania): Giorgia Rubulotta, Antonella Mo; Azienda Ospedaliera per l'Emergenza Cannizzaro-Terapia Intensiva Post Operatoria (Catania): Amalia Corso, Salvatore Girianni; Azienda Ospedaliera Universitaria Mater Domini (Catanzaro): Andrea Bruni, Eugenio Garofalo; SS. Annunziata Hospital (Chieti): Salvatore Maurizio Maggiore, Alessandro Di Risio; Ospedale San Giuseppe (Empoli): Italo Calamai, Rosario Spina; Arcispedale Sant'Anna Ferrara (Ferrara): Savino Spadaro, Carlo Alberto Volta; University Hospital O.O.R.R. (Foggia): Antonella Cotoia, Lucia Mirabella; Ospedale Civile (Imperia): Laura Maulicino, Giancarlo Abregal; Ospedale Madonna Delle Grazie (Matera): Maria Donvito, Paolo D’Ambrosio; Fondazione IRCCS Ca' Granda Ospedale Maggiore Policlinico Neurorianimazione (Milan): Filippo Binda, Ileana Adamina; Fondazione IRCCS Ca' Granda Ospedale Maggiore Policlinico Terapia Intensiva Generale E Vecla (Milan): Alessandro Galazzi; San Raffaele Hospital (Milan): Alessandra Negro; Azienda Ospedaliero-Universitaria Maggiore della Carità (Novara): Rosanna Vaschetto, Fabio Capuzzi; Azienda Ospedaliera di Padova (Padova): Margherita Boschetto, Lucia Stivanello; A.O.U. Policlinico Paolo Giaccone di Palermo Cardiothoracic ICU (Palermo): Luciano Bonaccorso, Chiara Megna; A.O.U. Policlinico Paolo Giaccone di Palermo UTIP (Palermo): Andrea Cortegiani, Pasquale Iozzo; P.O. Villa Sofia (Palermo): Antonino Rizzo, Giovanni Scire; Ospedali Riuniti Villa Sofia - Cervello General Intensive Care Unit (Palermo): Maria Rosa Taibi, Francesca Paola Tranello; Ospedali Riuniti Villa Sofia - Cervello Unità Operativa di 
Anestesia e Rianimazione I (Palermo): Antonio Manzo, Lidia Traina; Fondazione IRCCS Policlinico San Matteo (Pavia): Beatrice Pastore, Attilio Quaini; Azienda Ospedaliera Universitaria di Perugia (Perugia): Gian Domenico Giusti, Gloria Montaldi; USL Umbria 1 (Perugia): Federica Piergentili; Azienda Ospedaliera Santa Maria Della Misericordia (Perugia): Federica Mancini, Simona Casaioli; Azienda Ospedaliero Universitaria Pisana (Pisa): Francesco Uccelli, Fabio Guarracino; Fondazione Policlinico Agostino Gemelli TIPO (Rome): Adriana Onelli, Valentina Di Gravio; Policlinico Agostino Gemelli ICU (Rome): Maria Cossu, Oliva Matrona; Ospedale Sant'Andrea (Rome): Monica Rocco, Daniela Alampi; IRCCS Policlinico San Donato (San Donato Milanese): Federica Dellafiore, Flavia Ranalli; ASL CN1-SS. Annunziata Hospital (Savigliano): Matteo Bossolasco, Elisabetta Brizio; ASST Nord Milano po Sesto San Giovanni (Sesto San Giovanni): Pina Migliorino, Paolo Cortellazi; Azienda Ospedaliera Universitaria Senese (Siena): Moris Rosati, Francesco D’Ambrosio; Azienda ULSS2 Marca Trevigiana-Distretto Di Treviso (Treviso): Catia Quagliotto; Azienda Ospedaliera Universitaria di Trieste (Trieste): Erik Roman-Pognuz, Alberto Peratoner; San Bortolo Hospital (Vicenza): Silvia De Rosa, Marina Alessandra Martin; Ospedale Belcolle (Viterbo): Francesca De Sanctis, Paolo Ciorba.

JAMAICA: UHWI (Kingston): Kelly Fletcher, Patrick Toppin, Hyacinth Harding-Goldson 10/18.

JAPAN: Hiroshima University Hospital (Hiroshima): Shunsuke Taito, Nobuaki Shime; Kameda Medical Center (Kamogawa City, Chiba): Ryohey Yamamoto, Fumiya Kanda; Kobe University Hospital (Kobe): Akemi Hirao, Moritoki Egi; Kyoto Prefectural University of Medicin Hospital (Kyoto): Ayako Noguchi, Satoru Hashimoto; National Center for Global Health and Medicine (Tokyo): Umeda Aya; University of Tsukuba Hospital (Tsukuba): Hideaki Sakuramoto, Akira Ohuchi; Tokyo Bay Urayasu Ichikawa Medical Center (Urayasu): Jun Kataoka, Kumi Maruyama; Okinawa Chubu Hospital (Uruma): Izumi Nakayama, Yoshimasa Nishime; Yamaguchi Prefectural Grand Medical Center (Yamaguchi): Koji Fujimoto, Kenji Takahashi; Yokohama City University Medical Center (Yokohama): Mayumi Tsujimoto, Masako Shimizu.

KENYA: Aga Khan University Hospital Nairobi (Nairobi): Wangari Waweru-Siika, Eunice Tole; The Nairobi Hospital (Nairobi): Malcolm C Correia.

REPUBLIC OF KOREA: Korea University Ansan Hospital (Ansan): Je Hyeong Kim; Hallym University Sacred Heart Hospital (Anyang): Sunghoon Park; Daegu Catholic University Hospital (Daegu): Kyung Chan Kim; Yeungnam University Medical Center (Daegu): Jonghyun Baek, Jung-Min Bae; ChungNam National University
(Daejeon): So Young Park; Hanyang University Guri Hospital (Guri-si): Tai Sun Park; Chonbuk National University Hospital (Jeonju): Heung Bum Lee, Seung Yong Park; CHA Bundang Medical Center, CHA University (Seongnam-si, Gyeonggi-do): Jisoo Park; Seoul National University Bundang Hospital (Seongnam-si, Gyeonggi-do): Lee Yeon-Joo, Cho Young-Jae; Seoul National University Hospital (Seoul): Sang-Miin Lee, Samsung Medical Center (Seoul): Kyeongman Jeon; Seoul St.Mary's Hospital (Seoul): Seok Chan Kim, Jongmin Lee; Konkuk University Hospital (Seoul): Hyun Keun Chee; ASAN Medical Center (Seoul): Jin Won Huh; Hallym University Kangnam Sacred Heart Hospital (Seoul): Yun Su Sim; National Medical Center (Seoul): Junghyun Kim; Sanggye Paik Hospital, Inje University College of Medicine (Seoul): Youjin Chang, Ulsan University Hospital (Ulsan): Jong-Joon Ahn, Byung Ju Kang; Yonsei University Wonju Severance Christian Hospital (Wonju, Gangwon-do): Won-Yeon Lee, Seok Jeong Lee.

KOSOVO: University Clinical Center of Kosova (Prishtinë): Burhan Hadri, Nehat Baftiu.

LATVIA: Liepaja Regional Hospital (Liepaja): Ivasr Krastins; Riga East University Hospital (Riga): Mihails Dolgusevs.

LEBANON: LAUMCRH (Beirut): Fayez François Abillama, Sonia Stiban; Saint George Hospital (Beirut): Michel El Feghaly; Mount Lebanon Hospital-Gharios Medical Center (Hazmiyeh): Elie Gharios, Marie Merheb.

LYBIA: Tripoli University Hospital MICU (Tripoli): Mohamed Benlamin; Tripoli University Hospital CCU (Tripoli): Ala Khaled, Wesal Ali Belkhair; Tripoli Central Hospital TRAUMA ICU (Tripoli): Majd Tabib; Tripoli Central Hospital SICU (Tripoli): Firas Ashour, Ahmed Elhadi; Oya Clinic (Tripoli): Osama Wanees Emhemid Tababa, Taha Khaled; Alkhums Hospital (Alkhums): Soad Imhmed R. Alkhumsi; Septimus Hospital (Alkhums): Abdualhamid I Alshrif; Al-Fawz Clinic (Tripoli): Ahmed Ali Aboufray, Aya Alabuzidi; Safwa Clinic (Tripoli): Ahmed Ramadan Triki, Mala Elgammudi; Elkhadra Hospital SICU (Tripoli): Hajer Ben Zahra; Elkhadra Hospital MICU (Tripoli): Enas Soula, Maram Milud Said Al-Alawi; Abo Selim Trauma Hospital (Tripoli): Hazem Ahmed, Mohamed Abdurazzag Ali Ghula;

LITHUANIA: Republican Vilnius University Hospital (Vilnius): Saulius Vosylius.

LUXEMBURG: Centre Hospitalier Emile Mayrish (Esch-Sur-Alzette): Lucie Mouton, Touraj Rastegar; Centre Hospitalier du Nord (Ettelbruck): Claude Sertznig, Gilles Martin; Hôpital Kirchberg (Luxembourg): Cyril Thix, Christian Theisen; Centre Hospitalier de Luxembourg (Luxembourg): Christian Ferretti, Fränk Gils; ZithaKlinik (Luxembourg): Marc Gallion. 
MALAYSIA: Hospital Sultanah Bahiyah (Alor Setar, Kedah): Asmah Zainudin; Selayang Hospital (Batu Caves): Laila Kamaliah Kamalul Bahrin; Kuala Lumpur Hospital (Kuala Lumpur): Shanti Rudra Deva; Hospital Sultan Ismail (Johor Bahru, Johor): Azmin Huda Abdul Rahim; Hospital Tengku Ampuan Rahimah (Klang, Selangor): Sherliza Wahab; Hospital Universiti Sains Malaysia (Kota Bharu): Mohd Zulfakar Mazlan, W Nazaruddin W Hassan; Hospital Raja Perempuan Zainab II (Kota Bharu, Kelantan): Wan Nasrudin Wan Ismail, Mohd Nazri Ali; Queen Elizabeth Hospital (Kota Kinabalu): Tien Meng Khoo; Hospital Kuala Krai (Kuala Krai, Kelatan): Noryani Mohd Samat; Tuanku Ampuan Najihah Kuala Pilah (Kuala Pilah Negeri Sembilan): Jenny May Geok Tong; Hospital Sultanah Nur Zahirah (Kuala Terengganu): Nik Azman Nik Adib; International Islamic University Medical Centre (Kuantan): Mohd Basri Mat Nor, Shanthi Ratnam; Hospital Melaka (Melaka): Nahla $11 / 18$.

Ismail; Sungai Buloh Hospital (Sungai Buloh): Shanthi Ratnam; Hospital Raja Permaisuri Bainun (Ipoh, Perak): Siti Rohayah Sulaiman, Kit Weng Foong; Sibu Hospital (Sibu, Sarawak): Anita Alias, Ngu Pei Hua.

MAURITIUS: Wellkin Hospital (Moka): Sundaresan Maiyalagan; Clinique Muller (Curepipe): Sundaresan Maiyalagan.

MEXICO: Lic. Benito Juarez (Juarez. Chihuahua): Jorge Macias Zermeno; UMAE Instituto Mexicano del Seguro Social (Merida, Yacatan): Daniel Blanco; Unidad Médica Dede Alta Especialidad Ignacio Garcia Tellez (Merida, Yacatan): Karely Duran; Hospital General Zona 48 (Mexico): Claudia Lizbeth Lopez Nava, San Juan Roman Nandyelly; Hospital Especialidades Antonio Fraga Mouret (Mexico): Luis Alejandro Sanchez-Hurtado, Brigitte Tejeda-Huezo; Hospital General r. Gaudencio González Garza UMAE CMN La Raza IMSS (Mexico): Mario Del Moral Armengol, Luis Pedro Ambriz Nava; Hospital General Regional No.1 Carlos MacGregor Sánchez Navarro (Mexico): Jorge Guerra Herrera; Hospital de Especialidades del Centeo Medico Nacional Siglo XXI IMSS (Mexico): Gilberto Felipe Vazquez de Anda, Humberto Gallegos-Perez; Hospital de Traumatologia Dr. Victorio de la Fuente Narváez (Mexico): Nancy Hernandez-Sanchez, Lucia Hernandez-Ponce; Hospital Juárez de México (Mexico): Luis Gorordo-Delsol, Marcos Hernandez-Romero; Hospital General Dr Ruben Leñero (Mexico): Saira Gomez, Fernando Molinar; Hospital Médica Sur (Mexico): Silvio A. Namendys-Silva, Juan P. RomeroGonzalez; Hospital General de Zona 1A (Mexico): Daira Gonzalez; Hospital de Traumatología y Ortopedia 21IMSS (Monterrey): Antonio Landaverde, Miguel Ángel Sosa; Hospital General Regional Orizaba No 1 (Orizaba): Berenice Navarro; Clínica Mexico (Piedras Negras): José
Ivan Rodriguez de Molina Serrano; Hospital Regional ISSSTE Puebla (Puebla): Sergio Reyes Iburrigarro; Hospital General de Querétaro (Querétaro): Alejandro Ibarra, Joaquin Aguirre; Hospital General de Zona con Medicina Familiar No2 Francisco Padrón Puyou (San Luis Potosí): Mayra Martinez-Gonzalez; Hospital General Regional 180 (Tlajomulco de Zuñiga): Nayeli Rocio Cañas Padilla, Ana Alícia Velarde Pineda; Instituto Mexicano del Seguro Social HGZ No 1 (Tlaxcala): Missael Vladimir Espinoza Villafuerte, María Ocotlan Gonzalez Herrera.

MOLDOVA: Institute of Emergency Medicine (Chisinau): Adrian Belii.

MONGOLIA: First Central Hospital of Mongolia (Ulaanbaatar), National Trauma and Orthopaedic Research Hospital ((Ulaanbaatar), Third Central Hospital of Mongolia (Ulaanbaatar), Bayangol District Health Center (Ulaanbaatar), Central Military Hospital (Ulaanbaatar): Mendsaikhan Naranpurev, Battsetseg Baasanjav.

MOROCCO: Mohammed VI University Hospital (Marrakech): Abdelhamid Hachimi, Mina Elkhayari; Ibn Sina University Hospital (Rabat): Khalid Abidi, Tarek Dendane.

NEPAL: Chitwan Medical College Teaching Hospital (Bharatpur): Nisha Bhandari Subedi; Om Hospital and Research Center (Kathmandu): Sabina Dhakal Pathak; TU Teaching Hospital (Kathmandu): Prabha Gautam, Meena Manandhar.

NETHERLANDS: Meander MC (Amersfoort): Laura Van Gulik; Ziekenhuis Amstelland (Amstelveen): Mark Van Den Brink, Samantha Splinter Haaglanden Medical Center (The Hague): Peter Van Vliet, Benjamin Gerretsen; Haga Ziekenhuis (The Hague): Lettie Van Den Berg; Nij Smellinghe (Drachten): Marina De Haan, Binny Tuinstra; Catharina Ziekenhuis (Eindhoven): Paul Kuijpers, Jennifer Reijntjens; Medisch Spectrum Twente (Enschede): Jan Wytze Vermeijden, Martin Rinket; Rivas Zorggroep Beatrix Ziekenhuis (Gorinchem): Margijske Vanroest; Martiniziekenhuis (Groningen): Auke Reidinga, Bert Loef; University Medical Center Groningen (Groningen): Willem Dieperink, Marisa Onrust; Zuyderland Medical Center (Heerlen): Tom Dormans, Laura Bormans; Medical Centre Leeuwarden (Leeuwarden): Matty Koopmans, Rik T Gerritsen; St. Antonius Ziekenhuis (Nieuwegein): Arlette Van Den Elst; Canisius Wilhelmina Ziekenhuis (Nijmegen): Mirjam Evers, Oscar Oiting; Laurentius Hospital (Roermond): Rob Wilting; Bernhoven Ziekenhuis (Uden): Bart Ramaker, Mark van der Kuil; Diakonessenhuis (Utrecht): Jan-Willem Fijen, Lenneke Haas; UMC Utrecht (Utrecht): Dylan De Lange; Isala Ziekenhuis (Zwolle): Jasper Haringman.

NEW ZEALAND: Auckland City Hospital-DCCM (Auckland): Lynette Newby; Auckland City HospitalC\&V ICU (Auckland): Rachael Parke, Eileen Gilder; 
North Shore Hospital (Auckland): Danielle Hacking, Rica Dagooc; Middlemore Hospital (Auckland): Rima Song; Dunedin Hospital (Dunedin): Hansjoerg Waibel, Frances Dawn; Hawke's Bay Fallen Soldiers' Memorial Hospital (Hastings): Jackie Rapley, Llesley Chadwick; Hutt Valley DHB (Lower Hutt): Carmel Chapman; Nelson Hospital (Nelson): Petra Crone; Taranaki Base Hospital (New Plymouth): Jonathan Albrett, Peter Marko; Tauranga $12 / 18$

Hospital (Tauranga): Jennifer Goodson, Troy Browne; Timaru Hospital (Timaru): Richard Whitticase; Wellington Hospital (Wellington): Cheryl Davidson, Harriet Judd; Whagarei Base Hospital (Whangarei): Daniel Owens.

NIGERIA: College of Medicine, University of Nigeria Teaching Hospita (Enugu): Tonia Onyeka, Innocent Ugwu; University of Ibadan College of Medcine Hospital (Ibadan): Rose Ilesanmi, Prisca Olabisi Adejumo; Obafemi Awolowo University Teaching Hospitals Complex (Ile-Ife): Afolabi Owojuyigbe, Anthony Adenekan; Plateau State Specialist Hospital (Jos): Stella Uba, Christiana Chime; Jos University Teaching Hospital (Juth): Deborah Jibrin, Babangida John Sankey; Lagos University Teaching Hospital (Lagos): Oyebola Adekola, Simeon Olanipekun; Onelife Hospital (Lagos): Simeon Olanipekun, Oyebola Adekola.

NORTH MACEDONIA: University Clinic of Surgery Ss. Naum Ohridski (Skopje): Mirjana Shosolcheva, Vanja Gievski; University Hospital - TOARILUC Central ICU (Skopje): Andrijan Kartalov, Filip Naumovski; University Hospital-TOARILUC Neurosurgical ICU (Skopje): Biljana Kuzmanovska, Angela Trposak; Speclized Hospital for Surgical Diseases Zan Mitrev (Skopje): Zaneta Bogoevska-Miteva, Rodney Rosalia.

NORWAY: Østfold Hospital Trust (Grålum): Brita Fosser Olsen; Haukeland University Hospital SICU (Bergen): Britt Sjobo; Haukeland University Hospital MICU (Bergen): Karianne Dale Jensen, Drammen Sykehus, Vestre Viken HF GICU (Drammen): Birgitte Fosser Johansen; Drammen Sykehus, Vestre Viken HF ICU (Drammen): Esben Straede, Edda Johansen; Bærum Sykehus (Gjettum): Inger Johanne Finnstrom; UNN Harstad (Harstad): Annette Toellefsen, Haugesund Sykehus (Haugesund): Hege Ostenjo; Ringerike Sykehus Vestre Viken HF (Honefoss): Hege Bjorgen, Bjorn Bratsberg; Vestre Viken, Kongsberg Sykehus (Kongsberg): Elin Kristoffersen; Sørlandet Sykehus (Kristiansand): Elin Mari Skorstad; Akershus University Hospital (Lørenskog): Siri Hansen, Sylvi Vullum; Oslo University Hospital Thoraxkirurgisk Intensiv (Oslo): Gro Anne Lunde, Wenche Arntsen; Oslo University Hospital Generell intensiv Ullevål, $\mathrm{PO}$ /intensivavdelingen (Oslo): Mette Lund; Oslo University Hospital Generell Intensive 1 (Oslo): Gro Ringstad Akselsen; Oslo
University Hospital Generell Intensiv 2 (Oslo): Kristina Reinertsen Monstad; Oslo University Hospital Medisinsk Intensiv (Oslo): Ane Stenset; Oslo University Hospital Postoperativ 1 (Oslo): Hanne Haugom; Oslo University Hospital Neuro Intensive Care Unit (INTI3) (Oslo): Bjoern Monsen, Lisa Høgvall; Oslo University Hospital Hjertemedisinsk Intensiv og Overvåkning (Oslo): Siw Trudvang; Sykehuset Telemark (Skien): Britt Galaaen; Stavanger University Hospital (Stavanger): Siv Karin Malmin, Marit Hildegunn Andersen; Vestfold Hospital Trust (Tønsberg): Rita Foss Hargott, Yvonne Andersen; University Hospital of North Norway (UNN) (Tromso): Elin Steffenak, Marit Nyhus; St.Olavs Hospital (Trondheim): Barbro Meland.

PAKISTAN: Aga Khan University Hospital (Karachi): Madiha Hashmi.

PARAGUAY: Hospital de Trauma Manuel Giagni (Asunción): Noelia Rivas, Elizabeth Maidana; Instituto Nacional de Enfermedades Respiratorias y del Ambiente-INERAM (Asuncion): Alberto de Jesús Ortiz, Dolly Mabel Bordon Cabral; Sanatorio Adventista de Asuncion (Asuncion): Marcelo Simi, CesarAponte; Instituto Nacional de Cardiologia-Hospital San Jorge (Asuncion): Juan Carlos Rivas, Sirley Gill; Hospital Central IPS (Asuncion): Amilcar Garcia, Gloria Alvarenga; Hospital Regional Caacupe (Caacupe): Laura Cespedes, Hugo Perez; Hospital Fundacion Tesai (Ciudad del Este): Maria Liz Moreira, Fidelina Canete; Hospital Regional de Encarnacion (Encarnación): Roberto Gonzalez, Natalia Monges; Sanatorio San Sebastian (Fernando de la Mora): Amilcar Garcia, Mary Coman; Hospital de Clinicas-Facultad de Ciencias Medicas-UNA (San Lorenzo): Marcelo Pederzani, Natalia Franco.

PHILIPPINES: The Medical City Clark (Angeles City): Ferdinand Aganon, Regina Martinez; The Medical City Iloilo (Iloilo City): Debbie Noblezada-Uy; Ospital ng Muntinlupa (Muntinlupa): Chris Gerome Ellazar, Franklin Dean Cerezo; Asian Hospital and Medical Center (Muntinlupa,): Aaron Mark Hernandez; The Medical City (Pasig): Jose Emmanuel Palo, Cristal April Jane Aperocho; The Medical City South Luzon (Sta Rosa City): Michael Isanan.

POLAND: Szpital Uniwersytecki Nr 1 im.dr. A. Jurasza (Bydgoszcz): Marta Tubacka, Przemyslaw Jasiewicz; Medical University of Lublin First Public Teaching Hospital (Lublin): Miroslaw Czuczwar, Michal Borys, University Clinical Hospital (Olsztyn): Aleksandra Gutysz-Wojnicka, Lidia Glinka; Opole University Hospital (Opole): Ryszard Gawda; Infant Jesus Teaching Hospital (Warsaw): Malgorzata Mikaszewska-Sokolewicz; UCZKIN (Warsaw): Jan Bilawicz 13/18.

PORTUGAL: Unidade Local de Saúde do Baixo Alentejo, EPE-Hospital José Joaquim Fernandes 
(Beja): Paula Cabrita, João Vieira; Hospital Escala Braga (Braga): Margarida Ferreira Figueiredo, Cristiana Mota Pinheiro; Hospital Amato Lusitano- Unidade Local de Saúde de Castelo Branco (Castelo Branco): Nelson Antunes, Laura Pedro; Centro Hospitalar e Universitário de Coimbra-Polo HUC (Coimbra): Fatima Ferreira, Isabel Parente; Espirito Santo de Évora (Évora): Maria Varela; CHUA-Centro Hospitalar Universitário do Algarve (Faro): Fatima Fernandes, Claudia Martins; Hospital Dr. Nélio Mendonça (Funchal): Abel Viveiros; Hospital de São José UUM (Lisbon): Raquel Cavaco, Clara Santa Rita; Hospital de São José UCI (Lisbon): Sofia Dias, Ana Margarida Feranandes; Hospital de Santa Maria UCIMC (Lisbon): Pedro Silva; Hospital de Santa Maria SMI-UCIP (Lisbon): Catarina Nunes, Hospital de Santa Maria NUCI (Lisbon): João Cabral; Hospital de Cascais Dr.José de Almeida (Lisbon): Bruno Sousa, Filpe Pires; HOSPITAL Pr Dr Fernando Fonseca, EPE (Lisbon): Hilaryano Ferreira, Jacinta Santos; Hospital Beatriz Ângelo (Loures): Vitor Manuel Vaz Pinto, Bruno Miguel Bispo; Hospital Pedro HispanoUnidade Local de Saúde de Matosinhos (Matosinhos): Amelia Ferreira, Elena Molinos; CHTS (Penafiel): Estevão Lafuente; Hospital do Divino Espirito Santo (Ponta Delgada): Ricardo Gregorio, Humberto Costa; Centro Hospitalar Entre Douro e Vouga (Santa Maria da Feira): Ângela Lima, Susana Ferreira; Hospital do Litoral Alentejano (Santiago do Cacém): Vanda Seromenho, Eulália Luis; Centro Hospitalar de Setúbal (Setúbal): Idália Valerio; Hospital de Santarém (Santarém): Helena Cesar, Ana Tavares.

QATAR: Hamad Medical Corporation (Doha): Ahmed Subhy Alsheikhly, Saeed Mahmood.

ROMANIA: Prof. Dr. D. Gerota Hospital (Bucharest): Catalin Traian Guran, Alida Moise; Emergency Institute for Cardiovascular Diseases (Bucharest): Daniela Carmen Filipescu, Mihail Luchian; Fundeni Clinical Institute (Bucharest): Dana Tomescu, Mihai Popescu; Cluj University County Hospital (Cluj Napoca): Monica Adriana Scutariu, Clinical Emergency County Hospital Cluj (Cluj Napoca): Cristina Petrisor, Natalia Hagau; Regional Institute of Oncology(Iasi): Ioana Grigoras, Tatiana Patrichi.

RUSSIA: NMSC -State Budgetary Healthcare Institution, National Medical Surgical Center N.A. N.I. Pirogov, Ministry of Health of Russia (Moscow): Vitaly Gusarev, Alexandra Pivkina; GKB Muchina (Moscow): Vladimir Kulakov; Hospital SS Yudin (Moscow): Olga Ignatenko, Julia Kovaleva; Vishnevsky Institute of Surgery (Moscow): Trina Zhivotneva, Marina Zhedaeva; I.V. Davidovsky City Clinical Hospital (Moscow): Nikita Matiushkov; Burdenko Neurosurgery Institute (Moscow): Olga Ershova, Natalya Egorova; P.A. Herzen Moscow Cancer Research Institute (Moscow): Victoria Khoronenko, Danil
Baskakov; Research Center of Neurology (Moscow): Dmitry Sergeev, Michael Piradov; City Clinical Hospital №1 named after N.I. Pirogov (Moscow): Liudmila Grishina, Marat Magomedov; State Budgetary Healthcare Institution, National Medical Surgical Center n.a. N.I. Pirogov, Ministry of Health Of Russia CICU (Moscow): Evgeniy Zuev, Uri Gorokhovatsky; State Budgetary Healthcare institution, National Medical Surgical Center N.A.Pirogov,Ministry of Health of Russia ICU (Moscow): Anna Leonova, Liudmila Fadeeva; Privolzhskiy District Medical Center (Nizhniy Novgorod): Vladislav Belskiy, Dmitriy Galishevskiy; City Clinical Hospital\#4 (Perm): Nadezhda Zubareva, Maksim Tribulev; State Healthcare Institution of the Samara Region Clinical Hospital No. 5 (Toliyatti): Oksana Zueva, Alexander Kiselev; Tomsk National Research Medical Center of the Russian Academy of Sciences Cardiology Research Institute (Tomsk): Nikolaj Kamenshchikov, Ekaterina Tokareva; Tver Regional Hospital (Tver): Maxim Petrushin, Irina Starchenko.

RWANDA: Butare University Teaching Hospital CHU (Butare): Theogene Twagirumugabe, Isaac Nshimyumuremyi; Rwanda Military Hospital (Kigali): Jerome Muhizi, Egide Buregeya; Centre Hospitalier Universitaire de Kigali CHUK (Kigali): Josue Nzarora.

SAUDI ARABIA: ACH, King Khalid University (Abha): Amer Assiri, Ali Al Bshabshe Saudi German Hospital (Jeddah): Maie Salem Ebaid; Prince Sultan Military Medical City (Riyadh): Ghaleb Almekhlafi, Yasser Mandourah.

SERBIA: Clinical Center of Serbia-Clinic for Digestive Surgery (Belgrade): Jelena Velickovic, Dejan Veličković; Emergency centre, Clinical centre of Serbia ICU E (Belgrade): Bojan Jovanovic, Adi Hadzibegovic; Emergency Centre, Clinical Centre of Serbia ICU A (Belgrade): Branislava Stefanovic, Vanja Misic; Emergency center, clinical center of serbia ICU B (Belgrade): Vesna Bumbasirevic, Marija Rajković; Clinical Center Nis (Nis): Radmilo Jankovic, Milena Stojanovic; Institute for Pulmonary Diseases of Vojvodina (Sremska Kamenica): Srđan Gavrilovic, Maja Stanojević.

SLOVAKIA: UNB Hospital Ruzinov (Bratislava): Andrea Martonova, Aktham Yaghi; Faculty Hospital J.A. Reimana (Prešov): Anton Turčan, Peter Firment; NsP Revúca n.o. (Revúca): Garri Slobodianiuk; Faculty Hospital Trnava (Trnava): Daria Rabarova, Danca Lančaričová $14 / 18$.

SLOVENIA: General Hospital of Celje (Celje): Janko Vlaovic; University Medical Centre Ljubljana HDU Traumatology (Ljubljana): Matjaž Groznik; University Medical Centre Ljubljana ICU (Ljubljana): Milica Lukic, Janja Perme; University Medical Centre Ljubljana CICU (Ljubljana): Maja Sostaric, Nejc Umek; University Medical 
Centre Ljubljana SICU (Ljubljana): Tomislav Mirkovic, Simon Dolenc; University Medical Centre Ljubljana Center for Internal Intensive Medicine (Ljubljana): Rihard Knafelj, Misa Fister; UMC Ljubljana-Neurology Clinic (Ljubljana): Nika Zorko; (University Medical Centre Maribor (Maribor): Andrej Markota.

SOUTH AFRICA: King Edward VIII Hospital (Durban): Nomhle Princess Yeni, Phumele Jali; Charlotte Maxeke Johannesburg Academic Hospital (Johannesburg): Shelley Schmollgruber, Lenmed Royal Hospital and Heart Centre (Kimberley): Muhommed Ridwaan Syed; Edendale Hospital (Pietermaritzburg): Nivisha Parag, Robert Wise.

SPAIN: Hospital General Universitario de Alicante (Alicante): Maria Galiana, José Alejandro Navarro; Hospital Universitario del Sureste (Arganda del Rey): Ana María De Pablo, Patricia Albert; Hospital de Barbastro (Barbastro): Pilar Martinez, Yolanda Mendiara; Hospital del Mar (Barcelona): Barbara Garcia, Ana Alabart Llinas; Hospital Universitario Vall d'Hebron Traumatology and Burn ICU (Barcelona): Marilyn Riveiro; Hospital Universitario Vall d'Hebron ICU (Barcelona): Elisabet Gallart, Alba Riera; Hospital Clinic ICU (Barcelona): Miquel Sanz, Swagotika Salo; Hospital Clinic CICU (Barcelona): Miguel Angel Gimenez Lajara, Montserrat Venturas Nieto; Hospital Universitario Basurto Reanimacion (Basurto): Rosa Garcia, José Manuel Garcia Pena; Hospital Universitario Basurto Reanimacion Izda (Basurto): Maria Carmen Gorgolas, Maria Aranzazu Isasi; Hospital Universitario Puerta del Mar (Cadiz): Rafael Sierra; Hospital Universitario del Henares (Coslada): Federico Gordo, Isabel Conejo; Hospital de Dénia (Dénia): Vicent Salvà-Costa, Carolina Garzón-Tovar; Hospital Universitario de Fuenlabrada (Fuenlabrada): Sara Lospitao; Complejo Asistencial Universitario de León (León): Rafael Gonzalez, Pedro Gutierrez; Hospital Universitari de Bellvitge (L'Hospitalet del Llobregat): Mercè Girona, Jordi Adamuz; H.G.U Gregorio Marañón UCI (Madrid): Pablo Garcia Olivares, José Peral Gutierrez de Ceballos; H.G.U Gregorio Marañón UCP (Madrid): Celia Tirado, Irene De Wit; H.G.U Gregorio Marañón Reanimacion (Madrid): Ana Belén Curto Polo, Maria del Mar Diaz Salcedo; Infanta Leonor University Hospital (Madrid): Javier Ripolles-Melchor, Eugenio Martinez-Hurtado; Hospital Clínico San Carlos UCI SUR (Madrid): Jorge Duerto Alvarez, María Luisa Bravo Arcas; Hospital Clínico San Carlos UCI NORTE (Madrid): Juan Ignacio Torres Gonzalez, Ana Belén Sánchez de la Ventana; Hospital 12 Octubre UCI de Anestesia (Madrid): Pablo LopezArcas Calleja, Raquel Garcia Alvarez; Hospital Universitario Severo 0choa (Madrid): Purificacion Sanchez Zamora; Hospital Quironsalud Málaga (Malaga): Alvaro Ortega Guerrero; Fundació Althaia Xarxa Assistencial
Universitària de Manresa (Manresa): Rosario Cosano; Hospital Costa del Sol (Marbella): Jonathan PerezVacas, Margarita Campos-Perez; Consorci Sanitari del Maresme Hospital de Mataró (Mataró): Emma Moreno Barreiro, Losune Cano Sanchez; Hospital Universitario de Móstoles UCI (Móstoles): Monica Garcia Diaz; Hospital Universitario de Móstoles Reanimacion (Móstoles): Raquel Jimenez; Complexo Hospitalario Universitario de Ourense (Ourense): Lorena Del Rio Cabajo, Daniel Sancho Muriel; Hospital Universitario Central de Asturias UCI Polivalente (Oviedo): Helena Fernandez Alonso, Ana Wensell Fernández; Hospital Universitario Central de Asturias UCIC (Oviedo): Isabel Santín Piñan, Guillermo Muñiz Albaiceta; Hospital Universitario Central de Asturias Reanimación (Oviedo): Maria Cristina Iglesias Fernandez, Francisco Javier Saenz Abos; Clinica Universidad de Navarra (Pamplona): Pablo Monedero; Hospital Universitari Sant Joan (Reus): Ramon Molina Chueca, Lydia Gallego Aguirre; Parc Taulí Hospital Universitàri (Sabadell): Silvia Call Manosa, Carmen Partera Luque; Hospital Universitari General de Catalunya (Sant Cugat del Vallès): Neus Calpe, Monica Recio Losilla; Hospital Sant Joan Despí Moises Broggi (Sant Joan Despí): Meritxell Tapia Fores; Consorci Sanitari Garraf (Sant Pere de Ribes): Olga Farre, Oscar Fernandez; Hospital Universitario Santiago de Compostela (Santiago de Compostela): M. del Rosario Villar Redondo; Clínica HLA Santa Isabel (Sevilla): Donaldo S. Arteta Arteta, Maria Angeles Hurtado Sanchez; Sant Pau i Santa Tecla (Tarragona): Cristina Paños Espinosa, Laura Martinez Reyes; Hospital Universitari Joan XXIII (Tarragona): Laura Claramunt Domenech, Carmen Velasco Guillén; Hospital Universitari Mutua Terrassa (Terrassa): Josep Trenado Alvarez, Mercedes del Cotillo; Hospital Nacional de Parapléjicos (Toledo): Jesus Emilio Barrueco-Francioni, Belen Burgos Conde; Hospital Verge de la Cinta (Tortosa): Maria Pilar Sogues Blanco; Clinic University Hospital (Valencia): Maria Luisa Blasco, Ana Isabel Clement; Hospital Universitario Doctor Peset (Valencia): Clara Hurtado, Luz Coronado Sanz; Hospital Universitario Río Hortega (Valladolid): David Perez-Torres, Estefanía Prol-Silva; CHUVI- Alvaro Cunqueiro (Vigo): Jorge Pereira, Iván Areán González; Hospital Vithas San Jose Vitoria (Vitoria): Anastasio Espejo Cano; Hospital Universitario Araba (Txagorritxu) (Vitoria-Gasteiz): Cesar Rodriguez Nuñez, Inmaculada Lorenzo Fernadez; HUA Santiago Apostol. (Vitoria-Gasteiz): Alejandra Azahara 15/18.

Marguello Fernandez, Rosa Del Bosque Diez; Hospital de Zafra (Zafra): Badiola Hilario; Hospital Clinico Universitario Lozano-Blesa (Zaragoza): Begoña Zalba-Etayo, Ana Pascual-Bielsa.

SURINAME: s' Lands Hospitaal (Paramaribo): Bernardo Panka, Preveen Banwarie; St. Vincentius 
Ziekenhuis (Paramaribo): Dick Nahar, Alisha van Axel; Diakonessenhuis Hospital (Paramaribo): Naraindath N. Boedjawan.

SWEDEN: Sahlgrenska University Hospital TIVA (Goteborg): Erika Backlund Jansson, Ann-Sofie Malvemyr; Sahlgrenska University Hospital CIVA/96-NIVA (Goteborg): Lotta Johansson, Ulla Sandberg; Ryhov County Hospital (Jönköping): Catarina Tingsvik; Centralsjukhuset i Karlstad (Karlstad): Gunilla Mattsson, Gun Löf; Kristianstad Hospital (Kristianstad): Martin Spångfors; Kungälvs Hospital (Kungälv): Mona Ringdal; University Hospital (Linköping): Sebastian Geijer, Lotti Orvelius; Skane University Hospital (Malmö): Mia Hylen; Södersjukhuset AB MICU (Stockholm): Caroline Lagerhäll; Södersjukhuset IVA (Stockholm): Eva Joelsson-Alm; Eva-Marie Vesterlund-Rundgren; Karolinska University Hospital Funktionsområde Intensivvård (Stockholm): Eva Åkerman; Karolinska University Hospital NIVA (Stockholm): Viveca Hamback Hellkvist; Karolinska University Hospital PMI F21 (Stockholm): Ulrica Mickelsson, Eva Åkerman; Danderyds Hospital (Stockholm): Ewa Wahlbom; University Hospital (Uppsala): Ing-Marie Larsson, Ewa Wallin.

SWITZERLAND: Hôpitaux Universitaires de Genève (Genève): Filippo Boroli, Solenne Ory; Inselspital, Bern University Hospital (Bern): Joerg C. Schefold, Margaret Lynn Jong; Kantonsspital Frauenfeld (Frauenfeld): Alexander Dullenkopf, Martin Lang; Hopital Fribourgeois (Fribourg): Yvan Fleury, Marianne Maus; Lausanne University Hospital (CHUV) (Lausanne): Nawfel Ben-Hamouda, Anne Fishman.

TAIWAN: Hualien Tzu Chi, Buddhist Tzu Chi Medical Foundation (Hualien): Mei Yu Hsu, Shu Chuan Chang.

THAILAND: Faculty of Medicine Vajira Hospital Bangkok (Bangkok): Konlawij Trongtratul, Chaiwut Sawawiboon; Ramathibodi Hospital (Bangkok): Sunthiti Morakul; Songklanagarind Hospital (Hat Yai): Bodin Khwannimit.

TRINIDAD AND TOBAGO: San Fernando General Hospital (San Fernando): Lorna Merritt-Charles, Keevan Singh; Eric Williams Medical Sciences Complex (Champ Fleurs): Dale Ventour; Port of Spain General Hospital (Port Of Spain): Dianne Figaro-Barclay; Sangre Grande Hospital (Sangre Grande): Sasha Sankar-Maharaj.

TUNISIA: Mongi Slim University Hospital (La Marsa): Mhamed Sami Mebazaa, Salma Kamoun; Hôpital Taher Sfar Mahdia (Mahdia): Souheil Elatrous; Hopital universitaire Fattouma Bourguiba (Monastir): Lamia Besbes, Fekri Abroug; Hôpital Sahloul (Sousse): Walid Naija; Habib Thameur University Hospital (Tunis): Youssef Zied Elhechmi; Miltary Hospital (Tunis): Walid Sellami, Zied Hajjej; Zaghouan Regional Hospital (Zaghouan): Takoua Merhabene, Imen Talik.
TURKEY: Cukurova University Medical Faculty (Adana): Ozlem Ozkan Kuscu, Ozcengiz Dilek; Adana City Training and Research Hospital (Adana): Avşar Zerman; Turkey Advanced Speciality Education and Research Hospital (Ankara): Hayriye Cankar Dal, Sema Turan; Ankara Atatürk Göğüs Hastalıkları ve Gögüs Cerrahisi Eğitim ve Araştırma Hastanesi (Ankara): Semih Aydemir; UFUK University, Dr. Ridvan Ege Hospital (Ankara): Hakan Yilmaz; Ankara Yıldırım Beyazıt University Faculty of Medicine Atatürk Education and Research Hospital (Ankara): Duygu Kayar Calili, Seval İzdes; Akdeniz University Hospital (Antalya): Melike Cengiz, Ayça Gümüş; Adnan Menderes Üniversitesi Hastanesi (Aydın): Banu Taşdemir, Ali Kağnıcı; Batman Regional Hospital (Batman): Mustafa Ay, Serap Avcı Ay; Cekirge Public Hospital (Bursa): Gulbahar Caliskan; Duzce University, School of Medicine (Duzce): Turkay Akbas, Abidin Oner Balbay; Trakya University Medical Faculty Research Hospital (Edirne): Serdar Efe, Volkan Inal; Dr Ersin Arslan Eğitim Vve Araştırma (Gaziantep): Gülseren Elay; Isparta City Hospital (Isparta): Pınar Karabacak; Koç University Hospital (Istanbul): Boğaç Özserezli, Evren Şentürk; Istanbul University Cerrahpasa Medical School EICU (Istanbul): Oktay Demirkiran, Suha Bozbay; Istanbul University-Cerrahpasa, Cerrahpasa Medical School, Sadi Sun ICU (Istanbul): Yalım Dikmen, Elif Erdogan; University of Health Science Sisli Hamidiye Etfal Research and Training Hospital (Istanbul): Mustafa Akker, Nebia Peker; University of Health Sciences HNH Research and Training Hospital (Istanbul): Asu Ozgultekin; Umraniye Training and Research Hospital, University of Health Sciences, Interal Medicine ICU (Istanbul): Sibel Ocak Serin, Can Turan; Umraniye Training and Research Hospital, University of Health Science Anaesthesia and Reanimation (Istanbul): Gulsah Karaoren, Senay Goksu; Marmara Universitesi Egitim ve Arastirma Hastanesi MICU (Istanbul): Sait 16/18.

Karakurt, Huseyin Arikan; Marmara Universitesi Egitim ve Arastirma Hastanesi Anesthesiology and Reanimation ICU (Istanbul): Fethi Gül, İsmail Cinel; Konya Numune Hastanesi (Konya): Iskender Kara, Hasan Nabi Undar; Selcuk University, Faculty Of Medicine (Konya): Yesim Serife Bayraktar, Jale Bengi Çelik; Turkish Government Ministry Of Health Dumlupinar University Kutshya Education And Research Hospital (Kutayya): Murat Emre Tokur; Hafsa Sultan Hastanesi (Manesa): Demet Tok Aydin; Ordu University Medical Faculty Hospital (Ordu): İsmail Yildiz; Recep Tayyip Erdogan University Education and Research Hospital SICU (Rize): Beysim Özcan, Başar Erdivanli; Recep Tayyip Erdogan University Education and Research Hospital Anaesthesiology and Reanimation (Rize): Başar Erdivanli, Beysim Özcan; Karadeniz Technical University (Trabzon): Ahmet Eroglu; Adana Şehir 
Eğitim Araștırma Hastanesi (Yüreğir): Devrim Akdağ, Nurdan Ünlü.

UNITED ARAB EMIRATES: Amana Healthcare (Abu Dhabi): Mark Fielding, Adonis Dungca; Al Mafraq Hospital CICU (Abu Dhabi): Ashwaq Ali, Bindu Thankamma; Al Mafraq Hospital MICU (Abu Dhabi): Paul Eric Reyes, Sini John; Al Mafraq Hospital BICU (Abu Dhabi): Ajitha Rajendran; Al Mafraq Hospital SICU (Abu Dhabi): Fatima Kasem El Ahmad, Kathleen Ann Smiley; Sheikh Khalifa Medical City Ajman-General Hospita (Ajman): Susanna Hojden, Mia Thorning Miller; Al Ain Hospital (Al Ain): Vishnu Das Sasidharan Nair, Maria Gracia San Antonio; Tawam Hospital (Al Ain): Khaled Al Qawasmeh, Sabah Abu Shawish; Amana Medical and Rehabilitation Hospital (Al Ain): Hilary Twiggs, Ines Rosado; Kalba Hospital (Sharjah): Volodymyr Babych.

UNITED KINGDOM: Aberdeen Royal Infirmary (Aberdeen): Faye Morren; Buckinghamshire Health Care Trust (Aylesbury): Charlotte Young; Ysbyty Gwynedd (Bangor): Nicola Vaughan-Jones; North Devon District Hospital (Barnstaple): Stephanie Harris; Furness General Hospital (Barrow in Furness): Karen Burns; Basildon Hospital (Basildon): Carmel Georgiev, Rosina Shayamano; Royal United Hospital Bath NHSFT (Bath): Ian Kerslake, Peter Creber; Bedford Hospital NHS Trust (Bedford): Ana Vochin, Catherine O'Brien; Belfast Trust (Belfast): Paul Caddell; Ulster Hospital (Belfast): Samantha Hagan, Mandy Hughes; Queen Elizabeth Hospital Birmingham (Birmingham): Tomasz Torlinski, James Sherwin; City Hospital (Birmingham): Santhana Kannan, Amber Markham; Blackpool Teaching Hospitals NHS Foundation Trust (Blackpool): Richard Lebon, Jason Cupitt; Royal Bournemouth Hospital (Bournemouth): Julius Cranshaw, Nigel White; Bradford Teaching Hospital (Bradford): Victoria Marriott, Wendy Milner; Brighton and Sussex University Hospitals (Brighton): Casiano Barrera Groba; Cambridge University Hospitals NHS Foundation Trust (Cambridge): Joao Azoia, Petra Polgarova; Kent and Canterbury Hospital (Canterbury): Shaly George, Ritoo Kapoor; Royal Glamorgan Hospital (Cardiff): Ceri Lynch; Broomfield Hospital GICU (Chelmsford): Nathalie Fox, Karen Cranmer; Broomfield Hospital St Andrews Burns Unit (Chelmsford): Natalie Fox; Cheltenham General Hospital (Cheltenham): Thomas Llewellym, Kelly Matthews; St Peters Hospital (Chertsey): Louise Maltby, Jowena Ibao; Countess of Chester Hospital NHS Foundation Trust (Chester): Karen Boulton, Rachel Jarman; Colchester Hospital (Colchester): Karen Baxter; Croydon University Hospital (Croydon): Ashok Sundai Raj, Arif Moghal; Ysbyty Glan Clwyd (Denbighshire): Joanne White; Doncaster Royal Infirmary (Doncaster): Suzanne Barrowcliffe; Dorset County Hospital (Dorchester): Mark Pulletz, Vaarisan
Ganeshalingam; Western General Hospital (Edinburgh): Rosaleen Baruah; Royal Devon \& Exeter NHD Foundation Trust (Exeter): Carole Boulanger, Helen Baker; Frimley Park Hospital (Frimley): Justin Woods, Poe Poe Ei; Medway Maritime Hospital (Gillingham): Vongayi Ogbeide, Paul Hayden; Royal Gloucestershire Hospital (Gloucester): Kelly Matthews; Bolton Royal Hospital (Greater Manchester): Jennifer Hughes, Madhu Balasubramanian; Royal Surrey County Hospital (Guildford): Armorel Salberg; The Princess Alexandra Hospital NHS Trust (Harlow): Rajnish Saha, Dagmar Holmquist; Wycombe Hospital (High Wycombe): Charlotte Young; Calderdale \& Huddersfield NHS Foundation Trust (Huddersfield): Claire Derbyshire; Hull Royal Infirmary (Hull): Neil Smith, Elizabeth Stones; Ipswich Hospital NHS Trust (Ipswich): Jane Ademokun,; West Middlesex University Hospital (Isleworth): Monica Popescu, Maria Schofield Legorburo; Jersey General Hospital (Jersey): Samantha North, Carole Brett; Queen Elizabeth the Queen Mother Hospital (Kent): Helen Jaundoo; Royal Lancaster Infirmary (Lancaster): Jayne Craig; St James University Hospital (Leeds): Simon Whiteley; Leeds General Infirmary (Leeds): Clare Howcroft, Liz Wilby; Royal Liverpool University Hospital POCCU (Liverpool): Peter Delve, David Shaw; Royal Liverpool University Hospital ICU (Liverpool): Karen Williams, Ingeborg D Welters; Liverpool Heart and Chest Hospital (Liverpool): Jane McMullen; Hammersmith Hospital GICU (London): Stephen Brett, Leah Flores; Hammersmith Hospital CICU (London): Treiza Trueman-Dawkins; Charing Cross Hospital (London): Francesca Rubulotta, Mae Templeton; St Mary's Hospital (London): John Adams; The Royal London Hospital (London): Catherine Smith, John Prowle; St.Bartholomew's Hospital (London): Heather Byers, Andrea McDonnell; University Hospital Lewisham (London): Bernd Oliver Rose, Rosie Reece-Anthony; Kings's College Hospital NHS Foundation Trust (London): Luis Mendes; Chelsea and Westminster 17/18.

Hospital (London): Marcela Vizcaychipi, Rhian Bull; St George's University Hospitals NHS Foundation Trust (GICU, London): Grace Lacaden, Eleanor Santiago; St George's University Hospitals NHS Foundation Trust (CTICU, NICU, London): Carlos Castro Delgado, Sarah Farnell-Ward; University College Hospital (London): Elaine Thorpe; Macclesfield District General Hospital (Macclesfield): Justine Somerville, Anne Williams; Manchester Royal Infirmary (Manchester): Donna Cummings, Helen Derrick; Freeman Hospital (Newcastle): Sarah Brumwell, Claire Randell; Royal Victoria Infirmary Ward 18 (Newcastle Upon Tyne): Nicola McCann, Emma Aves; Royal Victoria Infirmary Ward 38 ITU (Newcastle Upon Tyne): Gillian Berry; Royal Gwent Hospital (Newport): Tamas Szakmany, Una 
Gunter; Kings Mill Hospital (Nottingham): Paul Pulak; Nottingham University Hospitals NHS Trust (Nottingham): Nikki Sarkar, Kerry Wright; The Manor Hospital (Oxford): Vitor Gomes; Tunbridge Wells Hospital (Pembury): Jo Jones, Ruth Palfrey; Poole Hospital NHS FT (Poole): Julie Camsooksai; Queen Alexandra Hospital (Portsmouth): Abby Lewis, Antony Eneas; St Helens \& Knowsley Teaching Hospitals NHS Trust (Prescot): Ascanio Tridente, Louise Barr; Queen's Hospital (Romford): Tomas Jovaisa, Beverley Thomas; Salford Royal NHS Foundation Trust (Salford): Emma Parkin, Daniel Horner; (South Tyneside District Hospital (South Shields): Christian Frey; Royal National Orthopaedic Hospital (Stanmore): Suzanne Bench, Rachel Baumber; Stepping Hill Hospital (Stockport): Phil Broadhurst, Matthew Jackson; North Tees \& Hartlepool NHS Foundation Trust (Stockton on Tees): Lynne Williams, Michele Clark; Royal Cornwall Hospital Trust (Truro): Jonathan Paddle, Sarah Bean; Mid Yorkshire Hospital (Wakefield): Sarah Buckley, Christopher Palfreeman; Warrington Hospital (Warrington): Sophie Liu, Nicola Allison; Warwick Hospital (Warwick): Ben Attwood, Penny Parsons; West Hertfordshire Hospitals Trust (Watford): Victoria Houghton, Sarah Jane Turner; Southend University Hospital (Westcliff on Sea): David Higgins, Egidija Bielskute; Royal Albert Edward Infirmary (Wigan): Nicola Horrigan; Wirral University Teaching Hospital (Arrowe Park Hospital) (Wirral): Reni Jacob, Karen Habgood; Queen Elizabeth Hospital (Woolwich): Ahmed Zaki, Amy Collins; Worthing Hospital (Worthing): Jenny Lord, Charalice Ramiro; Yeovil District Hospital (Yeovil): Agnieszka Kubisz-Pudelko, Michelle Kotze; Wrexham Maelor Hospital (Wrexham): Helen Williams.

UKRAINE: Mechnikov Dnipropetrovsk Regional Clinical Hospital (Dnipro): Ihor Iovenko, Alexander Tsarev; Kyiv Emergency Hospital (Kyiv): Lesia Zgrzheblovska.

URUGUAY: Hospital de Clinicas UdelaR (Montevideo): Arturo Briva; Gabriela Mendez.

UNITED STATES: University of Michigan Hospital ICU (Ann Arbor): Lena Napolitano; University of Michigan NICU CVICU (Ann Arbor): Leif Saager, Magnus Teig; Massachusetts General Hospital (Boston): Jarone Lee, Gabriel Enrique Rodriguez; Cooper University Hospital (Camden): Talia Ben-Jacob, Christopher Potestio; University of Virginia Medical Center (Charlottesville): Timothy Eng, Dea Mahanes; Cleveland Clinic Foundation (Cleveland): Ashish Khanna, Abhijit Duggal; Henry Ford Hospital (Detroit): Masakatsu Nananmori; JPS Health Network (Fort Worth): Manuel Lois, Anna Diaz; Penn State Health Milton S. Hershey Medical Center (Hershey): Kunal Karamchandani, Cheryl Bealer;
Houston Methodist San Jacinto Hospital (Houston): Cindy Barefield, Dorothy Terry, Pauline Fivecoat; University of Texas MD Anderson Cancer Center (Houston): Olakunle Idowu, Juan Cata; Ochsner Medical Center (Jefferson): Tara Clesi, Jessica Peterson; University of Kentucky Chandler Medical Center (Lexington): Kevin Hatton, Jasdeep Dhaliwal; Vanderbilt University Medical Center (Nashville): Dorothee Mueller; Yale New Haven Hospital (New Haven): Jing Tao, Ashley Szabo Eltorai; Memorial Sloan Kettering Cancer Center (New York): Stephen M Pastores, Natalie Remor; Rapid City Hospital (Rapid City): Janel Salazar, Javier Bogarin; Santa Barbara Cottage Hospital (Santa Barbara): Diane Barkas; Harborview Medical Center (Seattle): Aaron Joffe, Christopher Barnes; The University of Washington Medical Center (Seattle): Christopher Barnes; Barnes Jewish Hospital (St. Louis): Carrie Sona, Marilyn Schallom; Stanford University Hospital (Stanford): Jack Short, Javier Lorenzo.

VENEZUELA: Hospital Miguel Perez Catteño (Caracas): Ingrid Von Der Osten.

\section{Educative module development}

Marta Borkowska (European Society of Intensive Care Medicine, Brussels).

\section{Educative module content validation}

Mireia Llaurado-Serra (Nursing Department, Universitat Internacional de Catalunya, Barcelona, Spain), Liesbet Demarré (Nursing Staff, AZ Sint-Elisabeth, Zottegem, Belgium), Vincianne Pleitinckx (Clinical Advisor, Arjo Belgium) 18/18.

\section{Protocol translations}

Chinese: Frances Lin (Griffith University, Australia); Cynthia Xing (Mater Hospital Brisbane, Queensland, Australia), Jing Li (Peking University First Hospital, China).

French: Anne-Sophie Debue (Intensive Care Unit, Cochin Hospital Assistance Publique des Hôpitaux de Paris, France).

German: Stefan Goller (Department of Intensive Care Medicine, Inselspital, Bern, Switzerland.

Italian: Andrea Cortegiani (Section of Anesthesia, Analgesia Intensive Care and Emergency Department of Surgical, Oncological and Oral Science Policlinico Paolo Giaccone University of Palermo, Palermo, Italy), Chiara Megna (Cardiothoracic ICU, A.O.U. Policlinico Paolo Giaccone di Palermo, Palermo, Italy).

Portuguese: Elsa Afonso (Neonatal Intensive Care Unit, Rosie Maternity, Cambridge University Hospitals NHS Trust, Cambridge, United Kingdom; Ghent University, Faculty of Medicine \& Health Science, Dept. of Internal Medicine, Ghent, Belgium). 
Russian: V. Gusarov, Eugeniya Larina (State Budgetary Healthcare Institution, National Medical Surgical Center n.a. N.I. Pirogov, Ministry of Health of Russia, Moscow, Russia).

Spanish: M. Llaurado-Serra (Nursing Department, Universitat Internacional de Catalunya, Barcelona, Spain).

Thai: Konlawij Trongtrakul (Faculty of Medicine, Vajira Hospital, Navamindradhiraj University, Bangkok, Thailand).

Turkish: Yalim Dikmen, Elif Erdogan (Department of Intensive Care, Cerrahpasa Medical School, Istanbul University-Cerrahpasa, Turkey).

Open Access This article is licensed under a Creative Commons Attribution-NonCommercial 4.0 International License, which permits any non-commercial use, sharing, adaptation, distribution and reproduction in any medium or format, as long as you give appropriate credit to the original author(s) and the source, provide a link to the Creative Commons licence, and indicate if changes were made. The images or other third party material in this article are included in the article's Creative Commons licence, unless indicated otherwise in a credit line to the material. If material is not included in the article's Creative Commons licence and your intended use is not permitted by statutory regulation or exceeds the permitted use, you will need to obtain permission directly from the copyright holder. To view a copy of this licence, visit http://creativecommons.org/licenses/by-nc/4.0/.

Funding This project received funding from the European Society of Intensive Care Medicine (ESICM), the Flemish Society for Critical Care Nurses, and the HOGENT Fund for Applied Research. SB holds a research mandate from the Special Research Fund at Ghent University. In the UK, infrastructure support was provided by the National Institute for Health Research (NIHR) Imperial Bio-medical Research Centre (BRC). The views expressed are those of the authors and not necessarily those of the NHS, the NIHR or the Department of Health and Social Care. LR was funded by a TD Nursing Professorship in Critical Care Research from
Sunnybrook Research Institute, Toronto, Canada. The ESICM financed and co-administered the online data collection platform, provided a study webpage, and supported study administration. The other funding sources had no role in this work.

\begin{abstract}
Author details
${ }^{1}$ Nursing Department, Faculty of Education, Health and Social Work, HOGENT University of Applied Sciences and Arts, Ghent, Belgium. ${ }^{2}$ Department

of Internal Medicine, Faculty of Medicine and Health Science, Ghent University, C. Heymanslaan 10, 9000 Ghent, Belgium. ${ }^{3}$ Neonatal Intensive Care Unit, Rosie Maternity, Cambridge University Hospitals NHS Trust, Cambridge, UK. ${ }^{4}$ Hadassah Hebrew University Medical Center, Jerusalem, Israel. ${ }^{5}$ Wellcome-Wolfson Institute for Experimental Medicine, School of Medicine, Dentistry and Biomedical Sciences, Queen's University Belfast, Belfast, Northern Ireland, UK.

${ }^{6}$ Intensive Care Department, Royal Devon and Exeter NHS Foundation Trust, Exeter, Devon, UK. ${ }^{7}$ Section of Anaesthetics, Pain Medicine and Intensive Care, Department of Surgery and Cancer, Imperial College London, London, UK. ${ }^{8}$ Medical Intensive Care Unit, University Hospital of Grenoble-Alpes, Grenoble, France. ${ }^{9}$ Menzies Health Institute Queensland, School of Nursing and Midwifery, Griffith University, Brisbane, Australia. ${ }^{10}$ School of Nursing, Midwifery and Paramedicine, University of the Sunshine Coast, Sunshine Coast, QLD, Australia. ${ }^{11}$ School of Nursing, Royal Brisbane and Women's Hospital, Queensland University of Technology and Intensive Care Services (ICS), Herston, Australia.

${ }^{12}$ Institute for Skin Integrity and Infection Prevention, University of Huddersfield, Huddersfield, UK. ${ }^{13}$ Strategic Policy Cell, Ghent University Hospital, Ghent, Belgium. ${ }^{14}$ Division of Scientific Affairs-Research, European Society of Intensive Care Medicine, Brussels, Belgium. ${ }^{15}$ Intensive Care Department, CHU Brugmann University Hospital, Brussels, Belgium. ${ }^{16}$ Department for Anesthesia and Intensive Care, School of Medicine, University of Nis, Niš, Serbia.

${ }^{17}$ Section on Critical Care Medicine, Department of Anesthesiology, Critical IIIness, Injury Recovery and Research Center, Wake Forest School of Medicine, Winston-Salem, NC, USA. ${ }^{18}$ Outcomes Research Consortium, Cleveland, $\mathrm{OH}$, USA. ${ }^{19}$ Nursing Department, Universitat Internacional de Catalunya, BarceIona, Spain. ${ }^{20}$ Florence Nightingale Faculty of Nursing, Midwifery and Palliative Care, King's College London, London, UK. ${ }^{21}$ Sunnybrook Research Institute, Toronto, Canada. ${ }^{22}$ Lawrence S. Bloomberg Faculty of Nursing and Faculty of Medicine, University of Toronto, Toronto, Canada. ${ }^{23}$ Prolonged-Ventilation Weaning Centre, Michael Garron Hospital, Toronto, Canada. ${ }^{24}$ Klinik für Anaesthesiologie, Universitaetsmedizin Goettingen, Göttingen, Germany. ${ }^{25}$ Department of Anesthesiology, University of Michigan, Ann Arbor, USA.

${ }^{26}$ Al Mafraq Hospital, Abu Dhabi, United Arab Emirates. ${ }^{27}$ School of Nursing and Midwifery, Griffith University, Brisbane, Australia.
\end{abstract}

\section{Publisher's Note}

Springer Nature remains neutral with regard to jurisdictional claims in published maps and institutional affiliations.

Published online: 26 February 2021 